\title{
Calculation of thermal conductivity coefficients of electrons in magnetized dense matter
}

\author{
G. S. Bisnovatyi-Kogan \\ Space Research Institute, Rus. Acad. Sci., Profsoyuznaya str. 84/32, Moscow 117997, Russia. and \\ National Research Nuclear University MEPhI (Moscow Engineering Physics Institute), \\ Kashirskoye Shosse 31, Moscow, 115409, Russia. \\ M. V. Glushikhind \\ Space Research Institute, Rus. Acad. Sci., Profsoyuznaya str. 84/32, Moscow 117997, Russia.
}

(Dated: September 17, 2018)

\begin{abstract}
The solution of Boltzmann equation for plasma in magnetic field, with arbitrarily degenerate electrons and non-degenerate nuclei, is obtained by Chapman-Enskog method. Functions, generalizing Sonin polynomials are used for obtaining an approximate solution. Fully ionized plasma is considered. The tensor of the heat conductivity coefficients in non-quantized magnetic field is calculated. For non-degenerate and strongly degenerate plasma the asymptotic analytic formulas are obtained, which are compared with results of previous authors. The Lorentz approximation, with neglecting of electron-electron encounters, is asymptotically exact for strongly degenerate plasma.

We obtain, for the first time, in three polynomial approximation, with account of electron-electron collisions, analytical expressions for the heat conductivity tensor for non-degenerate electrons, in presence of a magnetic field. Account of the third polynomial improved substantially the precision of results. In two polynomial approximation our solution coincides with the published results.

For strongly degenerate electrons we obtain, for the first time, an asymptotically exact analytical solution for the heat conductivity tensor in presence of a magnetic field. This solution has considerably more complicated dependence on the magnetic field than those in previous publications, and gives several times smaller relative value of a thermal conductivity across the magnetic field at $\omega \tau \gtrsim 0.8$.
\end{abstract}

\section{INTRODUCTION}

Observations of thermal emission from neutron stars (NS) provides information about the magnetic field strength and configuration, temperature, chemical composition of the outer regions, and about the properties of matter at higher densities, deeper inside the star see [1], 2]. To derive this information, we need to calculate the structure and evolution of the star, and compare theoretical models with observational data. X-ray observations of thermal emission show periodic variabilities in single neutron stars [3], indicating to the anisotropic temperature distribution. It is produced at the low and intermediate density regions, such as the solid crust, where a complicated magnetic field geometry could cause a coupled magneto-thermal evolution. In some extreme cases, with a very high magnetic field, this anisotropy may even be present in the poorly known interior, where neutrino processes are responsible for the energy removal [4].

The spectrum of these NSs in broad range from optics to X-ray band, cannot be reproduced by a spectrum of the surface with a unique temperature. Fitting of the spectrum of the X-ray source $R X J 1856.53754$ in this broad region is explained [2] by a small hot emitting area $10-20 \mathrm{~km}^{2}$, and an extended cooler compo-

\footnotetext{
* gkogan@iki.rssi.ru

† m.glushikhina@iki.rssi.ru
}

nent. Another piece of evidence that strongly supports the nonuniform temperature distribution are pulsations in the X-ray signal of some objects of amplitudes $5-30 \%$, some of which have irregular light curves that point towards a non-dipolar temperature distribution [5].

Heat transfer in the envelopes of NS plays crucial role in many aspects of evolution of these stars. Thermal conductivity is the basic quantity needed for calculating the relationship between the internal temperature of a neutron star and its effective surface temperature. This relationship affects thermal evolution of the neutron star and its radiation spectra. To calculate thermal conductivity we should know the transport properties of a dense matter where electrons are degenerate, and form a nearly ideal Fermi-gas [6]. The ions are usually treated as nondegenerate. They may be in a gaseous state, may form a Coulomb liquid or a Coulomb crystal [7]. Under such conditions, electrons are the most important heat carriers, and the thermal conductivity is determined by electron motion. The magnetic field limits the motion of electrons in directions perpendicular to the field lines and, since they are the main carriers of the heat transport, the thermal conductivity in these directions is suppressed, while remaining unaffected along the field lines. The conductivity of electrons in NS and white dwarfs in presence of a magnetic field was studied in [8], [9]. The ratio between thermal conductivity along and across magnetic field lines considered in [8] was taken as 


$$
\frac{\lambda_{\perp}}{\lambda_{\|}}=\frac{1}{1+(\omega \tau)^{2}} .
$$

and was used also in [9]. Here $\omega$ is electron cyclotron frequency, $\tau$ is the time between collisions. The influence of the magnetic field on the electron heat conductivity in the form (11) was used in subsequent papers, see [10], 11]. Here we find an analytic solution for the heat conductivity tensor of strongly degenerate electrons in a magnetic field, in the Lorentz approximation, which is asymptotically exact in this case, showing a more complicated dependence on the magnetic field strength than (1).

Classical methods of kinetic gas theory were developed by Maxwell, Boltzmann, Gilbert, Enskog and Chapman. These methods are presented in the monograph of Chapman and Cowling [12]. They are based on the solution of the Boltzmann equation by method of successive approximations. As a zeroth approximation, the thermodynamic equilibrium distribution function is taken, which is a Maxwell distribution for a non-degenerate gas, and a Fermi-Dirac distribution in cases when degeneracy is important. The equilibrium distribution function is not an exact solution of Boltzmann equation in presence of non-uniformity. Following [12] we look for a solution of the Boltzmann equation in the first approximation by Sonyne (Laguerre) polynomial expansion, valid for a nondegenerate gas. To take into account a degeneracy, we use a set of orthogonal functions, as a generalization of Sonyne polynomials, suggested in [13], [14], [15], see also [16]. Only two first terms of this expansion are taken often for calculations of the heat conductivity coefficients. It was shown in [17] that this approximation gives substantial errors for the coefficient of a heat conductivity, which become much smaller when 3 polynomial expansion is used. Here we calculate the heat conductivity tensor in a magnetic field using 3 terms of the expansion. We show the improvement of the precision, when mowing from 2 to 3 polynomials, on the example of the non-magnetized Lorentz gas, where an exact solution is known.

The first application of the Boltzmann equation to the gas of charged particles was made by Chapman [12]. Due to the divergence of the collision integral at large impact parameters for particles with the Coulomb interaction, the upper limit of integration over the impact parameter was taken at the length of the average distance between particles. Thus the coefficients of viscosity, heat conductivity and diffusion of gases composed of charged particles were obtained. Divergence of the collision integral for Coulomb interaction at large impact parameter shows that the scattering with large impact parameters and small change of momentum in one collision play more important role than collisions with big change of the momentum. Landau used this fact to simplify the Boltzmann collision integral [18]. He expanded distribution function after collision in small variations of momentum and left first two terms. The integral obtained in this way is called Landau collision integral. Another derivation of the Landau collision integral was done by Chandrasekhar [19], who used the analog with the Brownian motion, described by the Fokker-Planck equation. The identity between Landau and Fokker-Planck collision integrals was shown in [20], see also [21].

The kinetic coefficients in the non-degenerate plasma, with and without magnetic field had been calculated in [22], 23], 24], 25] using Chapmen-Enskog expansion method. Braginskii 26] calculated the kinetic coefficients for a non-degenerate plasma in magnetic field consisting of electrons and one sort of positively charged ions, using kinetic equations normalized to average velocities, different for the ions and electrons. Landau collision integral was used, and two polynomials were taken into account in the expansion. The same approach, was used in [27], where calculations of kinetic coefficients for a fully ionized plasma of a complex composition have been performed. Coefficients of the heat conductivity tensor in a degenerate stellar cores were calculated in Lorentz approximation for a hydrogen plasma in [28]. A nonrelativistic calculation, based on on the quantum LenardBalescu transport equation for the thermal and electrical conductivity of plasma of highly degenerate, weakly coupled electrons and nondegenerate, weakly coupled ions was performed in [29].

Shear viscosities of non-relativistic, relativistic and ultra-relativistic hard sphere gas were calculated by Chapman-Enskog method in [30],31]

The present work is devoted to the solution of the Boltzmann equation by Chapman-Enskog method for electrons in an arbitrary degenerate plasma. We find a tensor of the heat conductivity using the expansion in two and three polynomials, and, on the example of the Lorentz gas, we show that the method has a good convergence to the exact solution.

We obtain, for the first time, in three polynomial approximation, with account of electron-electron collisions, analytical expressions for the heat conductivity tensor for non-degenerate electrons, in presence of a magnetic field. Account of the third polynomial improved substantially the precision of results. In two polynomial approximation our solution coincides with the published results.

For strongly degenerate electrons we obtain, for the first time, an asymptotically exact analytical solution for the heat conductivity tensor in presence of a magnetic field. This solution has considerably more complicated dependence on the magnetic field than those in previous publications, and gives several times smaller relative value of a thermal conductivity across the magnetic field at $\omega \tau \gtrsim 0.8$. 


\section{BOLTZMANN EQUATIONS AND TRANSFER EQUATIONS}

We use a Boltzmann equation for electrons, in a magnetic field, with an allowance of arbitrary degeneracy, and assuming them as non-relativistic We consider the electron gas in a crystal lattice of heavy nuclei, and take into account the interaction of the electrons with a nondegenerate nuclei and with one another. The nuclear component of the matter in the crust is in a crystal state, and therefore the isotropic part of the distribution function $f_{N 0}$ may differ from the Maxwellian distribution. If the mass of the nucleus $m_{N}$ is much greater, than the electron mass $m_{e}$, then to the terms $\sim m_{e} / m_{N}$ the details of the distribution function $f_{N 0}$ are unimportant, and the calculations can be made for arbitrary $f_{N 0}$.

Boltzmann equation, which describes the time variation of the electron distribution function $f$ in presence of the electric and magnetic fields is written as [24], 25]

$$
\frac{\partial f}{\partial t}+c_{i} \frac{\partial f}{\partial r_{i}}-\frac{e}{m_{e}}\left(E_{i}+\frac{1}{c} \varepsilon_{i k l} c_{k} B_{l}\right) \frac{\partial f}{\partial c_{i}}+J=0 .
$$

Here $(-e), m_{e}$ are the charge (negative) and the mass of the electron, $E_{i}, B_{i}$ are the strength of the electric field, and magnetic induction, $J$ is a collision integral, $\varepsilon_{i k l}$ is the totally antisymmetric Levi-Civita tensor, $c$ is the speed of the light.

$$
\begin{gathered}
J=J_{e e}+J_{e N}=R \int\left[f^{\prime} f_{1}^{\prime}(1-f)\left(1-f_{1}\right)-\right. \\
\left.-f f_{1}\left(1-f^{\prime}\right)\left(1-f_{1}^{\prime}\right)\right] \times g_{e e} b d b d \varepsilon d c_{1 i}+(3) \\
+\int\left[f^{\prime} f_{N}^{\prime}(1-f)-f f_{N}\left(1-f^{\prime}\right)\right] \times g_{e N} b d b d \varepsilon d c_{N i} .
\end{gathered}
$$

Here, the impact parameter $b$, and $\varepsilon$ are geometrical parameters of particle collisions with relative velocities

$$
g_{e e}, \quad g_{e N} ; \quad R=\frac{2 m_{e}^{3}}{h^{3}} .
$$

The integration in electron part of the collision integral in (3) is performed over the phase space of the incoming particles $\left(d c_{1 i}\right)$, and the physical space of their arrival $(b d b d \varepsilon)$ [12]. The velocity functions after collision are marked with touches.

The Boltzmann equation for electrons with a binary collision integral (3) may be applied in conditions, when the electron gas may be considered as almost ideal, i.e. the kinetic energy of the electrons is much larger than the energy of electrostatic interactions. It is valid for plasma at sufficiently small density. In the neutron stars and white dwarfs we have an opposite conditions of plasma at very large density, when it is important to take into account the electrons degeneracy. It is known from the statistical physics, that a gas of strongly degenerate electrons becomes ideal, because large Fermi energy substitutes here the thermal energy [32]. Therefore the calculations in this paper are applied to the low density, and high density plasma with degenerate electrons. Detailed discussion of the applicability of a binary collision integral (3), and its modifications for high density nondegenerate gases may be found in [12].

Lets introduce the thermal velocity of electrons, $v_{i}=$ $c_{i}-c_{0 i}$, where $c_{0 i}$ is the mass-average velocity. So we can write the Boltzmann equation with respect to the thermal velocity in the form [25]

$$
\begin{array}{r}
\frac{d f}{d t}+v_{i} \frac{\partial f}{\partial r_{i}}-\left[\frac{e}{m_{e}}\left(E_{i}+\frac{1}{c} \varepsilon_{i k l} v_{k} B_{l}\right)+\frac{d c_{0 i}}{d t}\right] \frac{\partial f}{\partial v_{i}} \\
-\frac{e}{m_{e} c} \varepsilon_{i k l} v_{k} B_{l} \frac{\partial f}{\partial v_{i}}-\frac{\partial f}{\partial v_{i}} v_{k} \frac{\partial c_{0 i}}{\partial r_{k}}+J=0,
\end{array}
$$

where

$$
\frac{d}{d t}=\frac{\partial}{\partial t}+c_{0 i} \frac{\partial}{\partial r_{i}}
$$

The transfer equations for the electron concentration, total momentum, and electron energy, in the twocomponent mixture of electrons and nuclei, can be obtained in a usual manner from the Boltzmann equation in a quasi-neutral plasma [12, 23 25] as

$$
\begin{gathered}
\frac{d n_{e}}{d t}+n_{e} \frac{\partial c_{0 i}}{\partial r_{i}}+\frac{\partial}{\partial r_{i}}\left(n_{e}\left\langle v_{i}\right\rangle\right)=0, \\
\rho \frac{d c_{0 i}}{d t}=\frac{1}{c} \varepsilon_{i k l} j_{k} B_{l}-\frac{\partial \Pi_{i k}}{\partial r_{k}}, \\
\frac{3}{2} k n_{e} \frac{d T}{d t}-\frac{3}{2} k T \frac{\partial}{\partial r_{i}}\left(n_{e}\left\langle v_{i}\right\rangle\right)+\frac{\partial q_{e i}}{\partial r_{i}}+\Pi_{i k}^{e} \frac{\partial c_{0 i}}{\partial r_{k}}= \\
=j_{i}\left(E_{i}+\frac{1}{c} \varepsilon_{i k l} c_{0 k} B_{l}\right)-\rho_{e}\left\langle v_{i}\right\rangle \frac{d c_{0 i}}{d t},
\end{gathered}
$$

where:

$$
\begin{gathered}
\Pi_{i k}=\sum_{\alpha} n_{\alpha} m_{\alpha}\left\langle v_{i}^{\alpha} v_{k}^{\alpha}\right\rangle, \quad \Pi_{i k}^{e}=n_{\alpha} m_{\alpha}\left\langle v_{i} v_{k}\right\rangle, \\
\left\langle v_{\alpha i}\right\rangle=\frac{R}{n_{\alpha}} \int f v_{\alpha i} d c_{\alpha i}, \quad n_{e}=R \int f d c_{e i} \\
c_{0 i}=\frac{1}{\rho} \sum_{\alpha} \rho_{\alpha}\left\langle c_{a i}\right\rangle, \quad j_{i}=-n_{e} e\left\langle v_{i}\right\rangle \\
q_{\alpha i}=\frac{1}{2} n_{\alpha} m_{\alpha}\left\langle v_{\alpha}^{2} v_{\alpha i}\right\rangle .
\end{gathered}
$$


Here summation is taken over the electrons and nuclei, $\Pi_{i k}^{e}=P_{e} \delta_{i k}, P_{e}=\frac{1}{3} n_{e} m_{e}\left\langle v^{2}\right\rangle$, when we neglect the electron viscosity, $P_{e}$ is the electron pressure, $\left\langle v_{i}\right\rangle$ is in average electron velocity in the comoving system, $q_{i}$ is the electron heat flux, and $j_{i}$ is the electron electric current. Here and in the subsequent consideration we identify the mass average velocity with the average velocity of ions $c_{0 i}=\left\langle c_{N} i\right\rangle$, and we consider the electric current and heat flux produced only by electrons. In the quasi neutral plasma the electron concentration $n_{e}$ is uniquely connected with the density $\rho$, defined by $\langle A, Z\rangle$ nuclei, $m_{N}=A m_{p}$

$$
\rho=m_{N} n_{N}, \quad n_{e}=\frac{Z \rho}{m_{N}} .
$$

\section{DERIVATION OF GENERAL EQUATIONS FOR THE FIRST APPROXIMATION FUNCTION}

The Boltzmann equation can be solved by ChapmenEnskog method of successive approximation [12]. This method is used here for conditions, when distribution functions are close to their values in thermodynamic equilibrium, and deviations are considered in a linear approximation. Equation for second order deviation from the equilibrium distribution function had been derived in 33. for a simple gas, see also 12]. The complexity of this equation, and rather narrow region where second order corrections could be important, strongly restricted the application of this approach.

The zeroth approximation to the electron distribution function is a Fermi-Dirac distribution, which is found by equating to zero of the collision integral $J_{e e}$ from (3)

$$
f_{0}=\left[1+\exp \left(\frac{m_{e} v^{2}-2 \mu}{2 k T}\right)\right]^{-1}, \quad R \int f_{0} d v_{i}=n_{e}
$$

Here, $\mu$ is a chemical potential of electrons, $k$ is Boltzmann's constant, $T$ is the temperature. The nuclear distribution function in the zeroth approximation $f_{N 0}$ is assumed to be isotropic with respect to the velocities and to depend on the local thermodynamic parameters; otherwise it can be arbitrary with the normalization:

$$
n_{N}=\int f_{N O} d c_{N i}
$$

where $n_{N}$ is the concentration of nuclei, $n_{e}=Z n_{N}, Z$ is the charge of the nucleus. Using (13) in (5)-(11), we obtain the zeroth approximation for the transfer equations. In this approximation $\left\langle v_{i}\right\rangle=0, q_{i}=0, \Pi_{i k}=$ $\left(P_{e}+P_{N}\right) \delta_{i k}$

$$
\begin{gathered}
n_{e}=2\left(\frac{2 k T m_{e}}{h^{2}}\right)^{3 / 2} G_{3 / 2}\left(x_{0}\right) \\
P_{e}=2 k T\left(\frac{2 k T m_{e}}{h^{2}}\right)^{3 / 2} G_{5 / 2}\left(x_{0}\right) \\
G_{n}\left(x_{0}\right)=\frac{1}{\Gamma(n)} \int_{0}^{\infty} \frac{x^{n-1} d x}{1+\exp \left(x-x_{0}\right)}, x_{0}=\frac{\mu}{k T}
\end{gathered}
$$

where $G_{n}\left(x_{0}\right)$ are Fermi integrals. In what follows, instead of $G_{n}\left(x_{0}\right)$ we will write $G_{n}$ cause the argument is the same. In the first approximation, we seek for the function $f$ in the form:

$$
f=f_{0}\left[1+\chi\left(1-f_{0}\right)\right]
$$

The distribution function $f_{N 0}$ is assumed to satisfy the relation:

$$
\frac{1}{n_{N}} \int v_{N i} v_{N k} f_{N 0} d c_{N i}=\delta_{i k} \frac{k T}{m_{N}} .
$$

The function $\chi$ admits representation of the solution in the form:

$$
\begin{gathered}
\chi=-A_{i} \frac{\partial \ln T}{\partial r_{i}}-n_{e} D_{i} d_{i} \frac{G_{5 / 2}}{G_{3 / 2}}, \\
d_{i}=\frac{\rho_{N}}{\rho} \frac{\partial \ln P_{e}}{\partial r_{i}}-\frac{\rho_{e}}{P_{e}} \frac{1}{\rho} \frac{\partial P_{N}}{\partial r_{i}}+\frac{e}{k T}\left(E_{i}+\frac{1}{c} \varepsilon_{i k l} c_{0 k} B_{l}\right) .
\end{gathered}
$$

The plasma is supposed to be quasineutral with a zero charge density. The functions $A_{i}$ and $D_{i}$ determine the heat transfer and diffusion. Substituting (19) in the equation for $\chi$ we obtain equations for $A_{i}, D_{i}[12]$. It was shown in [24], 25], that in presence of a magnetic field $B_{i}$, the polar vector $A_{i}$ (and similarly $D_{i}$ ) may be searched for in the form:

$$
A_{i}=A^{(1)} v_{i}+A^{(2)} \varepsilon_{i j k} v_{j} B_{k}+A^{(3)} B_{i}\left(v_{j} B_{j}\right)
$$

Introducing a function:

$$
\xi=A^{(1)}+i B A^{(2)},
$$

and dimensionless velocity: $u_{i}=\sqrt{\frac{m_{e}}{2 k T}} v_{i}$, we obtain the system for $\xi$ as 


$$
\begin{array}{r}
f_{0}\left(1-f_{0}\right)\left(u^{2}-\frac{5 G_{5 / 2}}{2 G_{3 / 2}}\right) u_{i}=\frac{i}{3} \frac{e m_{e} B}{\rho k T c} u_{i} f_{0}\left(1-f_{0}\right)\left[\int \xi_{N} f_{N 0} v_{N}^{2} d c_{N i}-R \int \xi f_{0}\left(1-f_{0}\right) v_{e}^{2} d c_{i}\right] \\
-i B f_{0}\left(1-f_{0}\right) \frac{e \xi}{m_{e} c} u_{i}+I_{e e}\left(\xi u_{i}\right)+I_{e N}\left(\xi_{N i} u_{N i}\right)
\end{array}
$$

where

and

$$
\begin{gathered}
I_{e e}\left(\xi u_{i}\right)=R \int f_{0} f_{01}\left(1-f_{0}^{\prime}\right)\left(1-f_{01}^{\prime}\right) \times\left(\xi u_{i}\right. \\
\left.+\xi_{1} u_{1 i}-\xi^{\prime} u_{i}^{\prime}-\xi_{1}^{\prime} u_{1 i}^{\prime}\right) g_{e e} b d b d \varepsilon d c_{1 i}, \\
I_{e N}\left(\xi u_{N i}\right)=\int f_{0} f_{N 0}\left(1-f_{0}^{\prime}\right)\left(\xi u_{i}\right. \\
\left.-\xi^{\prime} u_{i}^{\prime}\right) g_{e N} b d b d \varepsilon d c_{N i} .
\end{gathered}
$$

According to [12], a solution for the function $\xi$ is searched for in the form of the raw of orthogonal polynomials. Sonyne polynomial that were used in the classical work [12] are coefficients of the expansion of the function $(1-s)^{-\frac{3}{2}-1} e^{\frac{x s}{1-s}}$ in powers of $s$ :

$$
(1-s)^{-\frac{3}{2}-1} e^{\frac{x s}{1-s}}=\Sigma S_{3 / 2}^{(p)}(x) s^{p} .
$$

Sonyne polynomials are orthogonal:

$$
\int_{0}^{\infty} e^{-x} S_{3 / 2}^{(p)}(x) S_{3 / 2}^{(q)}(x) x^{3 / 2} d x=\frac{\Gamma\left(p+\frac{5}{2}\right)}{p !} \delta_{p q},
$$

$$
\begin{gathered}
S_{3 / 2}^{(0)}(x)=1, \quad S_{3 / 2}^{(1)}(x)=\frac{5}{2}-x, \\
S_{3 / 2}^{(2)}(x)=\frac{35}{8}-\frac{7}{2} x+\frac{1}{2} x^{2} .
\end{gathered}
$$

For a degenerate case we have to seek a solution of (23) in the form of an expansion in polynomials $Q_{n}$ that are orthogonal with the weight $f_{0}\left(1-f_{0}\right) x^{3 / 2}$, analogous to Sonyne polynomials [16].

$$
\begin{aligned}
& Q_{0}(x)=1, \quad Q_{1}(x)=\frac{5 G_{5 / 2}}{2 G_{3 / 2}}-x, \\
& Q_{2}(x)=\frac{35}{8} \frac{G_{7 / 2}}{G_{3 / 2}}-\frac{7 G_{7 / 2}}{2 G_{5 / 2}} x+\frac{1}{2} x^{2}, \quad x=u^{2} .
\end{aligned}
$$

The nonzero integrals of products of these polynomials with the corresponding weight function are

$$
\begin{gathered}
\int_{0}^{\infty} f_{0}\left(1-f_{0}\right) x^{3 / 2} Q_{0}^{2}(x) d x=\frac{3}{2} \Gamma(3 / 2) G_{3 / 2}\left(x_{0}\right), \\
\int_{0}^{\infty} f_{0}\left(1-f_{0}\right) x^{3 / 2} Q_{1}^{2}(x) d x=\frac{15}{4} \Gamma(3 / 2) G_{3 / 2}\left(x_{0}\right)\left(\frac{7}{2} \frac{G_{7 / 2}}{G_{5 / 2}}-\frac{5}{2} \frac{G_{5 / 2}^{2}}{G_{3 / 2}^{2}}\right), \\
\int_{0}^{\infty} f_{0}\left(1-f_{0}\right) x^{3 / 2} Q_{2}^{2}(x) d x=\frac{105}{16} \Gamma(3 / 2) G_{3 / 2}\left(x_{0}\right)\left(-\frac{35}{8} \frac{G_{7 / 2}^{2}}{G_{3 / 2}^{2}}+\frac{49}{2} \frac{G_{7 / 2}^{3}}{G_{5 / 2}^{2} G_{3 / 2}}-\frac{63}{2} \frac{G_{9 / 2} G_{7 / 2}}{G_{5 / 2} G_{3 / 2}}+\frac{99}{8} \frac{G_{11 / 2}}{G_{3 / 2}^{2}}\right) .
\end{gathered}
$$

We seek $\xi$ and $A_{3}$ in the form:

$$
\begin{array}{r}
\xi=a_{0} Q_{0}+a_{1} Q_{1}+a_{2} Q_{2}, \\
A^{(3)}=c_{0} Q_{0}+c_{1} Q_{1}+c_{2} Q_{2} .
\end{array}
$$

It is easy to show, with account of (16), (18), that the first term in the right side of (23) is $\sim \frac{m_{e}}{m_{N}}$ times smaller than the second one. Neglecting this term, multiplying (23) by $R Q_{0}(x) u_{i}, R Q_{1}(x) u_{i}$ and $R Q_{2}(x) u_{i}$ and integrating with respect to $d c_{i}$, we obtain a system of equation for the heat conductivity coefficients in the form 


$$
\left\{\begin{aligned}
& 0=-\frac{3}{2} i \omega n_{e} a_{0}+a_{0}\left(a_{00}+b_{00}\right)+a_{1}\left(a_{01}+b_{01}\right)+a_{2}\left(a_{02}+b_{02}\right) \\
&-\frac{15}{4} n_{e}\left(\frac{7 G_{7 / 2}}{2 G_{3 / 2}}-\frac{5 G_{5 / 2}^{2}}{2 G_{3 / 2}^{2}}\right)=-\frac{15}{4}\left(\frac{7 G_{7 / 2}}{2 G_{3 / 2}}-\frac{5 G_{5 / 2}^{2}}{2 G_{3 / 2}^{2}}\right) i \omega n_{e} a_{1}+a_{0}\left(a_{10}+b_{10}\right)+a_{1}\left(a_{11}+b_{11}\right)+a_{2}\left(a_{12}+b_{12}\right) \\
& 0=- \frac{105}{16}\left(-\frac{35}{8} \frac{G_{7 / 2}^{2}}{G_{3 / 2}^{2}}+\frac{49}{2} \frac{G_{7 / 2}^{2}}{G_{5 / 2}^{2}} \frac{G_{7 / 2}}{G_{3 / 2}}-\frac{63}{2} \frac{G_{9 / 2} G_{7 / 2}}{G_{5 / 2} G_{3 / 2}}+\frac{99}{8} \frac{G_{11 / 2}}{G_{3 / 2}}\right) i \omega n_{e} a_{2} \\
&+a_{0}\left(a_{20}+b_{20}\right)+a_{1}\left(a_{21}+b_{21}\right)+a_{2}\left(a_{22}+b_{22}\right)
\end{aligned}\right.
$$

Here $a_{j k}, b_{j k}$ are matrix elements for collision integrals, where $\omega=\frac{e B}{m_{e} c}$ is a cyclotron frequency.

\section{MATRIX ELEMENTS: $b_{j k}$}

The matrix elements, $b_{j k}$, connected with electronnuclei collisions, are determined as follows:

$$
\begin{array}{r}
b_{j k}=R \int f_{0} f_{N 0}\left(1-f_{0}^{\prime}\right) Q_{j}\left(u^{2}\right) u_{i}\left[Q_{k}\left(u^{2}\right) u_{i}\right. \\
\left.-Q_{k}\left(u^{\prime 2}\right) u_{i}^{\prime}\right] g_{e N} b d b d \varepsilon d c_{N i} d c_{i}, \\
k \geq 0 .
\end{array}
$$

Introduce functions $\bar{\Omega}_{e N}^{(l)}(r)$, defined as (see [12])

$\bar{\Omega}_{e N}^{(l)}(r)=2 \int_{0}^{\infty} f_{0}\left(1-f_{0}\right) z^{2 r+2} \int_{0}^{\infty}\left(1-\cos ^{l} \theta_{12}\right) g_{12} b d b d z$

$$
z=\left[\frac{m_{1} m_{2}}{2 k T\left(m_{1}+m_{2}\right)}\right]^{1 / 2} g_{12}, \quad g_{12}=\left|v_{1}-v_{2}\right|,
$$

for colliding particles "1", "2", $\theta_{12}$ is the scattering angle. At collisions of electrons ("2") and nuclei ("1") a mass of the nuclei is much greater than electron mass $m_{N} \gg m_{e}$, so we can neglect energy exchange in a collision

$$
u^{\prime 2} \approx u^{2}, \quad u u^{\prime} \approx u^{2}\left(1-\cos \theta_{12}\right), \quad g_{e N} \approx v, \quad z \approx u,
$$

Using the relation (14), we obtain from (33)

$$
b_{j k}=8 \pi^{2}\left(\frac{2 k T m_{e}}{h^{2}}\right)^{3 / 2}\left(\frac{2 k T}{m_{e}}\right)^{1 / 2} n_{N} \int_{0}^{\infty} f_{0}\left(1-f_{0}\right) Q_{j}(x) Q_{k}(x) x^{2} \int_{0}^{\infty}\left(1-\cos \theta_{12}\right) b d b d x
$$

Instead of functions $\bar{\Omega}_{e N}^{(l)}(r)$ from (34) we can use in this case functions $\widehat{\Omega}_{e N}^{(l)}(r)$ defined as

$$
\widehat{\Omega}_{e N}^{(l)}(r)=\int_{0}^{\infty} f_{0}\left(1-f_{0}\right) x^{r+1} \int_{0}^{\infty}\left(1-\cos ^{l} \theta_{12}\right) b d b d x .
$$

With account of (29), the elements of a symmetric matrix $b_{i j}$ are written as

$$
b_{00}=8 \pi^{2} \frac{(2 k T)^{2}}{h^{3}} m_{e} n_{N} \widehat{\Omega}_{e N}^{(1)}(1)
$$

$$
b_{01}=8 \pi^{2} \frac{(2 k T)^{2}}{h^{3}} m_{e} n_{N}\left(\frac{5}{2} \frac{G_{5 / 2}}{G_{3 / 2}} \widehat{\Omega}_{e N}^{(1)}(1)-\widehat{\Omega}_{e N}^{(1)}(2)\right),
$$




$$
\begin{gathered}
b_{11}=8 \pi^{2} \frac{(2 k T)^{2}}{h^{3}} m_{e} n_{N}\left(\frac{25}{4} \frac{G_{5 / 2}^{2}}{G_{3 / 2}^{2}} \widehat{\Omega}_{e N}^{(1)}(1)-5 \frac{G_{5 / 2}}{G_{3 / 2}} \widehat{\Omega}_{e N}^{(1)}(2)+\widehat{\Omega}_{e N}^{(1)}(3)\right) \\
b_{02}=8 \pi^{2} \frac{(2 k T)^{2}}{h^{3}} m_{e} n_{N}\left[\frac{35}{8} \frac{G_{7 / 2}}{G_{3 / 2}} \widehat{\Omega}_{e N}^{(1)}(1)-\frac{7}{2} \frac{G_{7 / 2}}{G_{5 / 2}} \widehat{\Omega}_{e N}^{(1)}(2)+\frac{1}{2} \widehat{\Omega}_{e N}^{(1)}(3)\right] \\
b_{12}=8 \pi^{2} \frac{(2 k T)^{2}}{h^{3}} m_{e} n_{N}\left[\frac{175}{16} \frac{G_{7 / 2} G_{5 / 2}}{G_{3 / 2}^{2}} \widehat{\Omega}_{e N}^{(1)}(1)-\frac{105}{8} \frac{G_{7 / 2}}{G_{3 / 2}} \widehat{\Omega}_{e N}^{(1)}(2)+\left(\frac{5}{4} \frac{G_{5 / 2}}{G_{3 / 2}}+\frac{7}{2} \frac{G_{7 / 2}}{G_{5 / 2}}\right) \widehat{\Omega}_{e N}^{(1)}(3)-\frac{1}{2} \widehat{\Omega}_{e N}^{(1)}(4)\right],(43) \\
\times\left[\frac{35^{2}}{8^{2}} \frac{G_{7 / 2}^{2}}{G_{3 / 2}^{2}} \widehat{\Omega}_{e N}^{(1)}(1)-\frac{245}{8} \frac{G_{7 / 2}^{2}}{G_{5 / 2} G_{3 / 2}} \widehat{\Omega}_{e N}^{(1)}(2)+\left(\frac{49}{4} \frac{G_{7 / 2}^{2}}{G_{5 / 2}^{2}}+\frac{35}{8} \frac{G_{7 / 2}}{G_{3 / 2}}\right) \widehat{\Omega}_{e N}^{(1)}(3)-\frac{7}{2} \frac{G_{7 / 2}}{G_{5 / 2}} \widehat{\Omega}_{e N}^{(1)}(4)+\frac{1}{4} \widehat{\Omega}_{e N}^{(1)}(5)\right]
\end{gathered}
$$

\section{A. Functions $\phi_{12}^{(l)}$, and Coulomb logarithm} form

The functions $\bar{\Omega}_{e N}^{(l)}(r)$ from (34) may be written in the

$$
\begin{aligned}
& \widehat{\Omega}_{e N}^{(l)}(r)=2\left[\frac{m_{1} m_{2}}{2 k T\left(m_{1}+m_{2}\right)}\right]^{1 / 2} \\
& \times \int_{0}^{\infty} f_{0}\left(1-f_{0}\right) z^{2 r+2} \phi_{12}^{(l)} d z
\end{aligned}
$$

with

$$
\phi_{12}^{(l)}=\int_{0}^{\infty}\left(1-\cos ^{l} \theta_{12}\right) g_{12} b d b .
$$

During the integration in (46) over the impact parameter $d b$ the integral has a logarithmic divergency at infinity. It is removed in a more precise treatment of Coulomb collisions in plasma with account of correlation functions [34], where the upper limit of the integration $b_{\max }$ appeared. Introducing $v_{0}=b g_{12}^{2}\left(m_{1} m_{2} / m_{0} e_{1} e_{2}\right)$, where $m_{0}=m_{1}+m_{2}, e_{1}, e_{2}$ are the absolute values of charges, we obtain after integration [12]

$$
\begin{gathered}
\phi_{12}^{(1)}=\left(\frac{m_{0} e_{1} e_{2}}{m_{1} m_{2}}\right)^{2} g_{12}^{-3} \ln \left(1+v_{0 \max }^{2}\right), \\
\phi_{12}^{(2)}=2\left(\frac{m_{0} e_{1} e_{2}}{m_{1} m_{2}}\right)^{2} g_{12}^{-3}\left[\ln \left(1+v_{0 \max }^{2}\right)-\frac{v_{0 \max }^{2}}{1+v_{0 \max }^{2}}\right],
\end{gathered}
$$

$$
v_{0 \max }=b_{\max } g_{12}^{2}\left(m_{1} m_{2} / m_{0} e_{1} e_{2}\right) .
$$

In farther integration the value under the logarithm is taken as constant when the average value $\bar{g}_{12}$ is taken instead of the variable $g_{12}$. For the electron-nuclei collisions with $g_{12} \approx v_{e}$ the approximate expression of the Coulomb logarithm is written in the form [35]

$$
\Lambda=\frac{1}{2} \ln \left(1+v_{0 \max }^{2}\right) \approx \bar{\Lambda}_{v}=\ln \left(\frac{b_{\max } \bar{v}_{e}^{2} m_{e}}{Z \mathrm{e}^{2}}\right), \quad \Lambda \gg 1,
$$

where

$$
\begin{aligned}
& \overline{v_{e}^{2}}= \frac{3 k T}{m_{e}} \frac{G_{5 / 2}}{G_{3 / 2}}=\frac{3 k T}{m_{e}} \quad(N D) \\
&=\frac{3}{5} \frac{h^{2}}{m_{\mathrm{e}}^{2}}\left(\frac{3 n_{e}}{8 \pi}\right)^{2 / 3} \quad(D) .
\end{aligned}
$$

The value of $b_{\max }$ is represented by the radius of Debye screening by electrons $r_{\mathcal{D} e}$, and ions $r_{\mathcal{D} i}$, and may be written as [35]

$$
\frac{1}{b_{\max }{ }^{2}}=\frac{1}{r_{\mathcal{D} i}^{2}}+\frac{1}{r_{\mathcal{D} e}^{2}}=\frac{4 \pi \mathrm{e}^{2}}{k T}\left(n_{N} Z^{2}+n_{e} \frac{G_{1 / 2}}{G_{3 / 2}}\right),
$$

where

$$
\frac{G_{1 / 2}}{G_{3 / 2}}=1
$$




$$
=4\left(3 \pi^{2}\right)^{1 / 3} \frac{m_{e} k T}{h^{2} n_{e}^{2 / 3}} \quad(D) .
$$

Influence of quantum effects on the Debye screening was discussed in [9]. The average frequency of electron-ion collisions $\nu_{e i}$ is written in [36] in the form

$$
\nu_{e i}=\frac{4}{3} \sqrt{\frac{2 \pi}{m_{e}}} \frac{Z^{2} e^{4} n_{N} \Lambda}{(k T)^{3 / 2} G_{3 / 2}} \frac{1}{1+e^{-x_{0}}} .
$$

In the limiting cases it is expressed as

$$
\begin{aligned}
\nu_{e i} & =\frac{4}{3} \sqrt{\frac{2 \pi}{m_{e}}} \frac{Z^{2} \mathrm{e}^{4} n_{N} \Lambda}{(k T)^{3 / 2}} \quad(N D) \\
& =\frac{32 \pi^{2}}{3} m_{e} \frac{Z^{2} \mathrm{e}^{4} \Lambda n_{N}}{h^{3} n_{\mathrm{e}}} \quad(D) .
\end{aligned}
$$

The average time $\tau_{e i}$ between (ei) collisions is the inverse value of $\nu_{e i}$, and is written as

$$
\begin{gathered}
\tau_{n d}=\frac{1}{\nu_{n d}}=\frac{3}{4} \sqrt{\frac{m_{e}}{2 \pi}} \frac{(k T)^{3 / 2}}{Z^{2} \mathrm{e}^{4} n_{N} \Lambda} \\
\tau_{d}=\frac{1}{\nu_{d}}=\frac{3 h^{3} n_{e}}{32 \pi^{2} m_{e} Z^{2} e^{4} \Lambda n_{N}} .
\end{gathered}
$$

\section{B. $b_{j k}$ for non-degenerate electrons}

For non-degenerate electrons we have $\exp \left(x-x_{0}\right) \ll$ $1, G_{n} \approx e^{x_{0}}$, so

$$
\widehat{\Omega}_{e N}^{(l)}(r)=e^{x_{0}} \int_{0}^{\infty} e^{-x} x^{r+1} \int_{0}^{\infty}\left(1-\cos ^{l} \theta\right) b d b d x .
$$

In this case it is more convenient to use functions $\Omega_{e N}^{(l)}(r)$ defined as

$$
\Omega_{e N}^{(l)}(r)=\frac{\sqrt{\pi}}{2} \int_{0}^{\infty} e^{-x} x^{r+1} \int_{0}^{\infty}\left(1-\cos ^{l} \theta\right) b d b d x .
$$

Using (45), (47) we find expressions for non-degenerate case as

$$
\begin{gathered}
\Omega_{e N}^{(1)}(r)=\sqrt{\pi} \frac{e^{4} \Lambda Z^{2}}{(2 k T)^{2}} \Gamma(r), \quad \Gamma(1)=1 ; \\
\Gamma(2)=1 ; \quad \Gamma(3)=2 ; \quad \Gamma(4)=6 ; \quad \Gamma(5)=24 .
\end{gathered}
$$

Substituting (60) into (39)-(44), taking into account that

$$
G_{n}=e^{x_{0}}, \quad e^{x_{0}}=\frac{n_{e}}{2 \pi^{3 / 2}}\left(\frac{h^{2}}{2 k T m_{e}}\right)^{3 / 2},
$$

and using (55), (56), we write $b_{j k}$ for non-degenerate electrons as

$$
b_{00}=8 \sqrt{\pi} \frac{n_{e} n_{N} e^{4} Z^{2} \Lambda}{(2 k T)^{3 / 2} \sqrt{m_{e}}}=\frac{3 n_{e}}{2 \tau_{n d}},
$$

$$
b_{01}=12 \sqrt{\pi} \frac{n_{e} n_{N} e^{4} Z^{2} \Lambda}{(2 k T)^{3 / 2} \sqrt{m_{e}}}=\frac{9 n_{e}}{4 \tau_{n d}},
$$

$$
b_{11}=26 \sqrt{\pi} \frac{n_{e} n_{N} e^{4} Z^{2} \Lambda}{(2 k T)^{3 / 2} \sqrt{m_{e}}}=\frac{39 n_{e}}{8 \tau_{n d}}
$$

$$
b_{02}=15 \sqrt{\pi} \frac{n_{e} n_{N} e^{4} Z^{2} \Lambda}{(2 k T)^{3 / 2} \sqrt{m_{e}}}=\frac{45 n_{e}}{16 \tau_{n d}}
$$

$$
\begin{gathered}
b_{12}=\frac{69 \sqrt{\pi}}{2} \frac{n_{e} n_{N} e^{4} Z^{2} \Lambda}{(2 k T)^{3 / 2} \sqrt{m_{e}}}=\frac{207 n_{e}}{32 \tau_{n d}}, \\
b_{22}=\frac{433 \sqrt{\pi}}{8} \frac{n_{e} n_{N} e^{4} Z^{2} \Lambda}{(2 k T)^{3 / 2} \sqrt{m_{e}}}=\frac{1299 n_{e}}{128 \tau_{n d}} .
\end{gathered}
$$

\section{C. $b_{j k}$ for partially degenerate electrons}

To calculate $b_{i j}$ for degenerate electrons we use expressions for $\widehat{\Omega}_{e N}^{(1)}(r)$ and $G_{n}\left(x_{0}\right)$. With account of (45), (47) we obtain

$$
\widehat{\Omega}_{e N}^{(1)}(r)=\int_{0}^{\infty} f_{0}\left(1-f_{0}\right) x^{r+1} \int_{0}^{b_{\max }}(1-\cos \theta) b d b d x=2 \frac{e^{4} Z^{2} \Lambda}{(2 k T)^{2}} \int_{0}^{\infty} f_{0}\left(1-f_{0}\right) x^{r-1} d x=2 \frac{e^{4} Z^{2} \Lambda}{(2 k T)^{2}} \Gamma(r) G_{r-1}\left(x_{0}\right)
$$

The integral in (68) is calculated exactly for $r=1$ $\int_{0}^{\infty} f_{0}\left(1-f_{0}\right) x^{r-1} d x=\left[1+\exp \left(-x_{0}\right)\right]^{-1}$. Between nondegenerate electrons with large negative non-dimensional 
chemical potential at $x_{0} \ll-1$, and strongly degenerate electrons with $x_{0} \gg 1$ there is a level of degeneracy at which $x_{0}=0$. Let us calculate matrix elements $b_{j k}$ for the level of degeneracy, corresponding to $x_{0}=0$. The functions $G_{n}(0)$ have the following numerical values, according to [32]

$$
G_{n}(0)=\frac{1}{\Gamma(n)} \int_{0}^{\infty} \frac{x^{n-1} d x}{1+e^{x}}=\left(1-2^{1-n}\right) \zeta(n),
$$

where Riemann $\zeta$-function has the following values for the indexes used here [37]

$$
\begin{gathered}
\zeta(3 / 2)=2.612, \quad \zeta(5 / 2)=1.341, \quad \zeta((7 / 2)=1.127 \\
\zeta(9 / 2)=1.0547, \quad \zeta(11 / 2)=1.0252, \quad \zeta(2)=1.645
\end{gathered}
$$

$$
\zeta(3)=1.202, \quad \zeta(4)=1.0823, \quad \zeta(5)=1.0369 .
$$

The functions $G_{n}(0)$ have the following values

$$
\begin{gathered}
G_{3 / 2}(0)=0.765, \quad G_{5 / 2}(0)=0.867, \quad G_{7 / 2}(0)=0.928, \\
G_{9 / 2}(0)=0.9615, \quad G_{11 / 2}=0.980, \quad G_{2}(0)=1.645, \\
G_{3}(0)=0.9015, \quad G_{4}(0)=0.947, \quad G_{5}(0)=0.972 .
\end{gathered}
$$

The functions $\widehat{\Omega}_{e N}^{(1)}(r)$ at $x_{0}=0$, defined as $\widehat{\Omega}_{e N 0}^{(1)}(r)$, have the following values, according to (68), using (69)-(71)

$$
\begin{gathered}
\widehat{\Omega}_{e N 0}^{(1)}(1)=\frac{e^{4} Z^{2} \Lambda}{(2 k T)^{2}} \equiv I, \quad \widehat{\Omega}_{e N 0}^{(1)}(2)=2 \ln 2 I=1.39 I \\
\widehat{\Omega}_{e N 0}^{(1)}(3)=3.29 I, \quad \widehat{\Omega}_{e N 0}^{(1)}(4)=10.82 I \\
\widehat{\Omega}_{e N 0}^{(1)}(5)=45.46 I .
\end{gathered}
$$

The level of degeneracy $D L(0)$ at $x_{0}=0$ is defined as a ratio of the Fermi energy $\varepsilon_{f e}$ to $k T$. With account of (15), (171), we obtain

$$
\begin{gathered}
D L(0)=\frac{\varepsilon_{f e}}{k T}=\frac{\left(3 \pi^{2} n_{e}\right)^{2 / 3} h^{2}}{8 \pi^{2} m_{e} k T} \\
=\frac{\pi}{4}\left(\frac{3}{\pi}\right)^{2 / 3}\left[2 G_{3 / 2}(0)\right]^{2 / 3}=1.011 .
\end{gathered}
$$

At $x_{0}=0$ the expression for the electron concentration $n_{e 0}$ from (15), and the average time $\tau_{e i}$ between (ei) collisions, which is the inverse value of $\nu_{e i}$, are written, using (54), (71), (16) as

$$
n_{e 0}=2 G_{3 / 2}(0)\left(\frac{k T m_{e}}{2 \pi \hbar^{2}}\right)^{3 / 2}=2 \times 0.765\left(\frac{k T m_{e}}{2 \pi \hbar^{2}}\right)^{3 / 2},
$$

$$
\tau_{d 0}=\frac{3}{4} \sqrt{\frac{m_{e}}{2 \pi}} \frac{(k T)^{3 / 2} G_{3 / 2}}{Z^{2} e^{4} n_{N} \Lambda}\left(1+e^{-x_{0}}\right)
$$

$$
=0.765 \frac{3}{2} \sqrt{\frac{m_{e}}{2 \pi}} \frac{(k T)^{3 / 2}}{Z^{2} e^{4} n_{N} \Lambda} .
$$

Using (72), (74, (75) we find from (39)-(44)

$$
\begin{gathered}
b_{00}=\frac{8 \pi^{2} e^{4} Z^{2} m_{e} \Lambda}{h^{3}} n_{N}=\frac{3}{2} \frac{n_{e 0}}{\tau_{d 0}} \\
b_{01}=2.16 \frac{n_{e 0}}{\tau_{d 0}}, \quad b_{11}=5.162 \frac{n_{e 0}}{\tau_{d 0}}, \quad b_{02}=2.588 \frac{n_{e 0}}{\tau_{d 0}} \\
b_{12}=6.671 \frac{n_{e 0}}{\tau_{d 0}}, \quad b_{22}=11.038 \frac{n_{e 0}}{\tau_{d 0}} .
\end{gathered}
$$

For arbitrary level of degeneracy at $x_{0} \neq 0$ the functions $G_{n}\left(x_{0}\right)$ in (16) are not expressed analytically, and should be calculated numerically for each $x_{0}$, at corresponding DL

$$
D L\left(x_{0}\right)=\frac{\left(3 \pi^{2} n_{e}\right)^{2 / 3} h^{2}}{8 \pi^{2} m_{e} k T}=\frac{\pi}{4}\left(\frac{3}{\pi}\right)^{2 / 3}\left[2 G_{3 / 2}\left(x_{0}\right)\right]^{2 / 3} .
$$

After numerical calculation of $G_{n}\left(x_{0}\right)$, the matrix elements $b_{j k}$ at arbitrary $x_{0}$ are found in the same way as it is done above at $x_{0}=0$.

\section{D. $b_{j k}$ for strongly degenerate electrons}

For strongly degenerate case $x_{0} \gg 1$ we use [32] the following expansions 


$$
G_{r}\left(x_{0}\right)=\frac{1}{\Gamma(r)}\left[\frac{x_{0}^{r}}{r}+\frac{\pi^{2}}{6}(r-1) x_{0}^{r-2}+\frac{7 \pi^{4}}{360}(r-1)(r-2)(r-3) x_{0}^{r-4}\right] \text { for } r \geq 1
$$

$$
\Gamma(r) G_{r-1}\left(x_{0}\right)=(r-1)\left[\frac{x_{0}^{r-1}}{r-1}+\frac{\pi^{2}}{6}(r-2) x_{0}^{r-3}+\frac{7 \pi^{4}}{360}(r-2)(r-3)(r-4) x_{0}^{r-5}\right] \text { for } r \geq 2
$$

For strongly degenerate electrons

$$
x_{0}=\frac{\left(3 \pi^{2} n_{e}\right)^{2 / 3} h^{2}}{8 \pi^{2} m_{e} k T} \gg 1
$$

We obtain than from (68), (81), omitting exponentially

$$
\widehat{\Omega}_{e N}^{(1)}(1)=2 \frac{e^{4} Z^{2} \Lambda}{(2 k T)^{2}} \int_{0}^{\infty} f_{0}\left(1-f_{0}\right) d x=2 \frac{e^{4} Z^{2} \Lambda}{(2 k T)^{2}}
$$

$$
\widehat{\Omega}_{e N}^{(1)}(2)=2 \frac{e^{4} Z^{2} \Lambda}{(2 k T)^{2}} \Gamma(2) G_{1}\left(x_{0}\right)=2 \frac{e^{4} Z^{2} \Lambda}{(2 k T)^{2}} x_{0},
$$
small terms $\sim e^{-x_{0}}$

$$
\begin{gathered}
\widehat{\Omega}_{e N}^{(1)}(3)=2 \frac{e^{4} Z^{2} \Lambda}{(2 k T)^{2}} \Gamma(3) G_{2}\left(x_{0}\right)=2 \frac{e^{4} Z^{2} \Lambda}{(2 k T)^{2}}\left(x_{0}^{2}+\frac{\pi^{2}}{3}\right), \\
\widehat{\Omega}_{e N}^{(1)}(4)=2 \frac{e^{4} Z^{2} \Lambda}{(2 k T)^{2}} \Gamma(4) G_{3}\left(x_{0}\right)=2 \frac{e^{4} Z^{2} \Lambda}{(2 k T)^{2}}\left(x_{0}^{3}+\pi^{2} x_{0}\right), \\
\widehat{\Omega}_{e N}^{(1)}(5)=2 \frac{e^{4} Z^{2} \Lambda}{(2 k T)^{2}} \Gamma(5) G_{4}\left(x_{0}\right)=2 \frac{e^{4} Z^{2} \Lambda}{(2 k T)^{2}}\left(x_{o}^{4}+2 \pi^{2} x_{0}^{2}+\frac{7 \pi^{2}}{15}\right) .
\end{gathered}
$$

Using (80), (83)- (87), we find from (39)-(44)

$$
b_{12}=\frac{709 \pi^{4} n_{e}}{960 x_{0} \tau_{d}}, \quad b_{22}=\frac{2 \pi^{4} n_{e}}{15 \tau_{d}}
$$

$$
b_{00}=\frac{16 \pi^{2} e^{4} Z^{2} m_{e} \Lambda}{h^{3}} n_{N}=\frac{3 n_{e}}{2 \tau_{d}},
$$

$$
b_{01}=\frac{3 \pi^{2} n_{e}}{4 x_{0} \tau_{d}}, \quad b_{11}=\frac{\pi^{2} n_{e}}{2 \tau_{d}}, \quad b_{02}=-\frac{7 \pi^{4} n_{e}}{320 x_{0}^{2} \tau_{d}}
$$

The matrix elements, $a_{j k}$, related to electron-electron collisions, are determined as follows:

$$
a_{j k}=R^{2} \int f_{0} f_{01}\left(1-f_{0}^{\prime}\right)\left(1-f_{01}^{\prime}\right) Q_{j}\left(u^{2}\right) u_{i}\left[Q_{k}\left(u^{2}\right) u_{i}+Q_{k}\left(u_{1}^{2}\right) u_{1 i}-Q_{k}\left(u^{\prime 2}\right) u_{i}^{\prime}-Q_{k}\left(u_{1}^{\prime}\right) u_{1 i}^{\prime}\right] g_{e e} b d b d \varepsilon d c_{1 i} d c_{i}
$$

Let's introduce following variables [12]

$$
\begin{gathered}
G_{l i}=\frac{1}{2}\left(c_{i}+c_{1 i}\right)=\frac{1}{2}\left(c_{i}^{\prime}+c_{1 i}^{\prime}\right), \\
g_{e e, i}=c_{1 i}-c_{i}, \quad g_{e e, i}^{\prime}=c_{1 i}^{\prime}-c_{i}^{\prime}, \\
g_{e e}=\left|g_{e e, i}\right|=\left|g_{e e, i}^{\prime}\right|=g_{e e}^{\prime}, \quad G_{0 i}=G_{l i}-c_{0 i}, \\
v_{i}=G_{0 i}-\frac{1}{2} g_{e e, i}, \quad v_{i 1}=G_{0 i}+\frac{1}{2} g_{e e, i},
\end{gathered}
$$

Here $G_{l i}$ is a velocity of the center of mass of two colliding electrons in the laboratory frame, $G_{0 i}$ is the same value in the comoving frame, $g_{e e, i}$ is a relative velocity of two colliding electrons before encounter, $g_{e e, i}^{\prime}$ is the 
same value after encounter; $v_{i}$ and $v_{1 i}$ are velocities of colliding electrons in the comoving frame, defined above. Introduce non-dimensional variables
Here $u_{i}, u_{1 i}$ are non-dimensional velocities of electrons, defined above. The matrix elements

$$
\begin{gathered}
g_{i}=\frac{1}{2}\left(\frac{m_{e}}{k T}\right)^{1 / 2} g_{e e, i}, \quad g_{i}^{\prime}=\frac{1}{2}\left(\frac{m_{e}}{k T}\right)^{1 / 2} g_{e e, i}^{\prime}, \\
g=\left|g_{i}\right|=\left|g_{i}^{\prime}\right|=g^{\prime}, \quad G_{i}=\left(\frac{m_{e}}{k T}\right)^{1 / 2} G_{0 i}, \\
d c_{i} d c_{1 i}=\left(\frac{2 k T}{m_{e}}\right)^{3} d G_{i} d g_{i}, \\
u^{2}+u_{1}^{2}=G^{2}+g^{2}, \quad u^{2}=u_{i}^{2}, \quad u_{1}^{2}=u_{1 i}^{2}, \quad G^{2}=G_{i}^{2} .
\end{gathered}
$$

$$
a_{j 0}=8\left(\frac{2 k T m_{e}}{h^{2}}\right)^{3}\left(\frac{k T}{m_{e}}\right)^{1 / 2} \int f_{0} f_{01}\left(1-f_{01}^{\prime}\right)\left(1-f_{0}^{\prime}\right) Q_{j}\left(u^{2}\right) u_{i}\left[u_{i}+u_{1 i}-u_{i}^{\prime}-u_{1 i}^{\prime}\right] g b d b d \varepsilon d g_{i} d G_{i}=0
$$

$a_{j 0}$ are equal to zero because the momentum conservation during encounter define the zero value in the brackets of
(94). The nonzero matrix elements $a_{j k}(j, k \geq 1)$ are defined as

$$
a_{j k}=8\left(\frac{2 k T m_{e}}{h^{2}}\right)^{3}\left(\frac{k T}{m_{e}}\right)^{1 / 2} \int f_{0} f_{01}\left(1-f_{01}^{\prime}\right)\left(1-f_{0}^{\prime}\right) Q_{j} u_{i}\left[Q_{k} u_{i}+Q_{k} u_{1 i}-Q_{k}^{\prime} u_{i}^{\prime}-Q_{k}^{\prime} u_{1 i}^{\prime}\right] g b d b d \varepsilon d g_{i} d G_{i}
$$

Here $Q_{i}$ are function of $u^{2}$ or $u_{1}^{2}$, and $Q_{i}^{\prime}$ are function of following expression $(j, k \geq 1)$ $u^{\prime 2}$ or $u_{1}^{\prime 2}$ respectively.

\section{A. $a_{j k}$ for non-degenerate electrons}

For non-degenerate case, at $x_{0} \gg 1, f_{0} \ll 1$, polynomials $Q_{i}$ are reduced to $S_{3 / 2}^{(i)}$, and we have from (95) the

$$
a_{j k}=8\left(\frac{2 k T m_{e}}{h^{2}}\right)^{3}\left(\frac{k T}{m_{e}}\right)^{1 / 2} e^{2 x_{0}} \int e^{-u^{2}-u^{\prime 2}} S_{3 / 2}^{(j)} u_{i}\left[S_{3 / 2}^{(k)} u_{i}+S_{3 / 2}^{(k)} u_{1 i}-S_{3 / 2}^{(k)^{\prime}} u_{i}^{\prime}-S_{3 / 2}^{(k)^{\prime}} u_{1 i}^{\prime}\right] g b d b d \varepsilon d g_{i} d G_{i}
$$

The integrals

$$
\left[S_{3 / 2}^{(j)}, S_{3 / 2}^{(k)}\right]=\frac{2}{\pi^{3}}\left(\frac{k T}{m_{e}}\right)^{1 / 2} \int e^{-u^{2}-u^{\prime 2}} S_{3 / 2}^{(j)} u_{i}\left[S_{3 / 2}^{(k)} u_{i}+S_{3 / 2}^{(k)} u_{1 i}-S_{3 / 2}^{(k)^{\prime}} u_{i}^{\prime}-S_{3 / 2}^{(k)^{\prime}} u_{1 i}^{\prime}\right] g b d b d \varepsilon d g_{i} d G_{i}
$$

are calculated in [12], and are defined by formulae

$$
\begin{gathered}
{\left[S_{3 / 2}^{(1)}, S_{3 / 2}^{(1)}\right]=4 \Omega_{e e}^{(2)}(2),} \\
{\left[S_{3 / 2}^{(1)}, S_{3 / 2}^{(2)}\right]=7 \Omega_{e e}^{(2)}(2)-2 \Omega_{e e}^{(2)}(3),} \\
{\left[S_{3 / 2}^{(2)}, S_{3 / 2}^{(2)}\right]=\frac{77}{4} \Omega_{e e}^{(2)}(2)-7 \Omega_{e e}^{(2)}(3)+\Omega_{e e}^{(2)}(4) .}
\end{gathered}
$$


The functions $\Omega_{e e}^{(l)}(r)$ are similar to functions (59), and are defined in [12] as

$$
\begin{gathered}
\Omega_{e e}^{(l)}(r)=\frac{\sqrt{\pi}}{2} \int_{0}^{\infty} e^{-x} x^{r+\frac{1}{2}} \phi_{e e}(l) d x, \\
\phi_{e e}(l)=\int_{0}^{\infty}\left(1-\cos ^{l} \theta\right) b d b, \quad x=g^{2} .
\end{gathered}
$$

Using (97), (98), (61) in (96), we have

$$
a_{j k}=n_{e}^{2}\left[S_{3 / 2}^{(j)}, S_{3 / 2}^{(k)}\right]
$$

For plasma with $\Lambda \gg 1$ from (50) we have from (48), (99)

$$
\begin{gathered}
\phi_{e e}(2) \approx \frac{16 e^{4}}{m_{e}^{2} g_{e e}^{3}}, \\
\Omega_{e e}^{(2)}(r)=\sqrt{\pi} \frac{e^{4} \Lambda}{\sqrt{m_{e}}(k T)^{3 / 2}} \Gamma(r) .
\end{gathered}
$$

Using (101), we have from (98), (100), with account of (56), with $n_{e}=Z n_{N}$

$$
\begin{gathered}
a_{11}=4 n_{e}^{2} \frac{\sqrt{\pi} \Lambda e^{4}}{\sqrt{m_{e}}(k T)^{3 / 2}}=\frac{3}{\sqrt{2}} \frac{n_{e}}{Z \tau_{n d}}, \\
a_{12}=3 n_{e}^{2} \frac{\sqrt{\pi} \Lambda e^{4}}{\sqrt{m_{e}}(k T)^{3 / 2}}=\frac{9}{4 \sqrt{2}} \frac{n_{e}}{Z \tau_{n d}}, \\
a_{22}=\frac{45}{4} n_{e}^{2} \frac{\sqrt{\pi} \Lambda e^{4}}{\sqrt{m_{e}}(k T)^{3 / 2}}=\frac{135}{16 \sqrt{2}} \frac{n_{e}}{Z \tau_{n d}} .
\end{gathered}
$$

\section{B. $a_{j k}$ for degenerate electrons}

The matrix elements $a_{j k}$ for strongly degenerate case had been found analytically in [15], see also [16]. They were calculated for strongly degenerate neutrons in a nuclear matter in [15], and for the neutrons in the inner crust of a neutron star, with many free neutrons [16]. It was found in the last case that in presence of nondegenerate heavy nuclei, and strongly degenerate neutron, the input of collisions between them in the heat transfer and diffusion coefficients is negligibly small, in comparison with neutron-nuclei collisions. The same situation we have for the strongly degenerate electrons, for which, using results of [15], the estimations give $a_{j k} \sim b_{j k} / x_{0}^{2} \ll b_{j k}$ for $x_{0} \gg 1$. Therefore for strongly degenerate electrons the Lorentz approximation, with account of collisions between light and heavy particles only, is asymptotically exact. The heat transfer coefficients for strongly degenerate electrons in presence of a magnetic field are calculated in section VIII.
The situation is more complicated for partially degenerate electrons. In this case there are no analytical expressions for the matrix elements $a_{j k}$, which should be found numerically by integration of multi-dimensional integrals in (95). Another problem is more serious. As shown on section VII, the precision of polynomial approximation is decreasing with increasing of the level of degeneracy. For non-degenerate electrons the result of three-polynomial approximation in Lorentz gas at $B=0$ is less than the exact result in Lorentz approximation by only about $2.2 \%$, see (145) and (146). Similar calculations for moderately degenerate electrons at $x_{0}=0$ in (152) and (153) show that the result of three polynomial approximation is about $87 \%$ of the exact result. Therefore for stronger degeneracy the result of 3-polynomial approximation will be even farther (less) from the exact result, and to obtain good results in the polynomial approximation the number of polynomials should increase with the level of degeneracy. That leads to very cumbersome analytical calculations. In looks out that it is better to solve this problem by numerical calculations, if a good precision is needed. In astrophysical problems it could be enough to use the interpolation formulae between sufficiently exact results obtained in 3-polynomial approximation for nondegenerate electrons with account of $e e$ collisions, and asymptotically exact result for strongly degenerate electrons in Lorentz approximation. The discussion of this problem is given in section IX.

\section{TENSOR OF A HEAT CONDUCTIVITY}

The heat flux is expressed via the heat conductivity tensor in the form [24, 25, 38]:

$$
q_{i}=-\lambda_{i k} \frac{\partial T}{\partial r_{k}}
$$

where $\lambda_{i k}$ :

$$
\begin{aligned}
\lambda_{i k}=\frac{5}{2} & \frac{k^{2} T n_{e}}{m_{e}} \frac{G_{5 / 2}}{G_{3 / 2}}\left\{\left[a_{0}^{1}-\left(\frac{7}{2} \frac{G_{7 / 2}}{G_{5 / 2}}-\frac{5}{2} \frac{G_{5 / 2}}{G_{3 / 2}}\right) a_{1}^{1}\right] \delta_{i k}\right. \\
- & \varepsilon_{i k n} B_{n}\left[b_{0}^{1}-\left(\frac{7}{2} \frac{G_{7 / 2}}{G_{5 / 2}}-\frac{5}{2} \frac{G_{5 / 2}}{G_{3 / 2}}\right) b_{1}^{1}\right] \quad(106) \\
& \left.+B_{i} B_{k}\left[c_{0}^{1}-\left(\frac{7}{2} \frac{G_{7 / 2}}{G_{5 / 2}}-\frac{5}{2} \frac{G_{5 / 2}}{G_{3 / 2}}\right) c_{1}^{1}\right]\right\}
\end{aligned}
$$

Here $a_{0}^{1}, a_{1}^{1}$; and $b_{0}^{1}, b_{1}^{1}$ are the real and imaginary parts of the coefficients $a_{0}$ and $a_{1}$, respectively:

$$
\begin{aligned}
a_{0} & =a_{0}^{1}+i B b_{0}^{1}, & a_{1} & =a_{1}^{1}+i B b_{1}^{1} \\
B^{2} c_{0}^{1} & =\left(a_{0}^{1}\right)_{B=0}-a_{0}^{1}, & B^{2} c_{1}^{1} & =\left(a_{1}^{1}\right)_{B=0}-a_{1}^{1}
\end{aligned}
$$


To find the coefficients $a_{0}, a_{1}$ for arbitrary electron degeneracy it is necessary to solve the system of equations (32) with matrix elements $b_{j k}$ from (39)-(44), and matrix elements $a_{j k}$ from section V. For arbitrary degeneracy of electrons the coefficients in the heat conductivity tensor, as well as well as in 3 other tensors defining the transport of a heat and electrical current in a dense plasma, may be evaluated only numerically. In two limiting cases of non-degenerate, and strongly degenerate electrons the results are found analytically.

\section{A. Heat conductivity tensor for non-degenerate electrons}

For non-degenerate electrons tensor (106) can be written as follows:

$$
\lambda_{i k}=\frac{5}{2} \frac{k^{2} T n_{e}}{m_{e}}\left[\left(a_{0}^{1}-a_{1}^{1}\right) \delta_{i k}-\varepsilon_{i k n} B_{n}\left(b_{0}^{1}-b_{1}^{1}\right)+B_{i} B_{k}\left(c_{0}^{1}-c_{1}^{1}\right)\right]
$$

The system for 3-polynomial solution for the electrons in presence of magnetic field, following from (32), with account of (62)-(67), (102)-(104), is written as

$$
\left\{\begin{array}{r}
0=-\frac{3}{2} i \omega \tau_{n d} a_{0}+\frac{3}{2} a_{0}+\frac{9}{4} a_{1}+\frac{45}{16} a_{2} \\
-\frac{15}{4} \tau_{n d}=-\frac{15}{4} i \omega \tau_{n d} a_{1}+\frac{9}{4} a_{0}+\frac{3}{2}\left(\frac{13}{4}+\frac{\sqrt{2}}{Z}\right) a_{1}+\frac{9}{8}\left(\frac{23}{4}+\frac{\sqrt{2}}{Z}\right) a_{2} \\
0=-\frac{105}{16} i \omega \tau_{n d} a_{2}+\frac{45}{16} a_{0}+\frac{9}{8}\left(\frac{23}{4}+\frac{\sqrt{2}}{Z}\right) a_{1}+\frac{3}{32}\left(\frac{433}{4}+\frac{45 \sqrt{2}}{Z}\right) a_{2}
\end{array}\right.
$$

Two first equations at $a_{2}=0$ determine the 2 -polynomial approximation, giving with account of (108) the following results for the case $B=0$

$$
\begin{aligned}
a_{0} & =\frac{15}{4} \frac{\tau_{n d}}{1+\frac{\sqrt{2}}{Z}}, \quad a_{1}=-\frac{5}{2} \frac{\tau_{n d}}{1+\frac{\sqrt{2}}{Z}}, \\
\lambda_{n d}^{(2)} & =\frac{125}{8} \frac{k^{2} T n_{e}}{m_{e}} \frac{\tau_{n d}}{1+\frac{\sqrt{2}}{Z}}=15.63 \frac{k^{2} T n_{e}}{m_{e}} \frac{\tau_{n d}}{1+\frac{\sqrt{2}}{Z}} .
\end{aligned}
$$

The above results coincide with the results obtained in [23], [24]. In 3-polynomial approximation we obtain the solution of (109) for $a_{0}, a_{1}$, and heat conductivity coefficient for the case $B=0$, with account of (108), as

$$
\begin{aligned}
& a_{0}=\frac{165}{32} \frac{1+\frac{15 \sqrt{2}}{11 Z}}{1+\frac{61 \sqrt{2}}{16 Z}+\frac{9}{2 Z^{2}}} \tau_{n d} \\
& a_{1}=-\frac{65}{8} \frac{1+\frac{45 \sqrt{2}}{52 Z}}{1+\frac{61 \sqrt{2}}{16 Z}+\frac{9}{2 Z^{2}}} \tau_{n d}
\end{aligned}
$$

$$
\begin{aligned}
\lambda_{n d}^{(3)} & =\frac{2125}{64} \frac{k^{2} T n_{e}}{m_{e}} \frac{1+\frac{18 \sqrt{2}}{17 Z}}{1+\frac{61 \sqrt{2}}{16 Z}+\frac{9}{2 Z^{2}}} \tau_{n d}, \\
& =33.20 \frac{k^{2} T n_{e}}{m_{e}} \frac{1+\frac{18 \sqrt{2}}{17 Z}}{1+\frac{61 \sqrt{2}}{16 Z}+\frac{9}{2 Z^{2}}} \tau_{n d},
\end{aligned}
$$

The value

$$
Q=\frac{64 m_{e} \lambda_{n d}^{(3)}}{2125 k^{2} T n_{e} \tau_{n d}}=\frac{1+\frac{18 \sqrt{2}}{17 Z}}{1+\frac{61 \sqrt{2}}{16 Z}+\frac{9}{2 Z^{2}}}
$$

showing how nondegenerate electron-electron collisions decrease the heat conductivity coefficient at $B=0$ is presented in Table 1 for different values of $Z$.

In two polynomial approximation, taking $a_{2}=0$, we obtain the solution of the system (109) in the form

$$
a_{0}=\frac{15}{4} \tau_{n d} \frac{1}{1+\frac{\sqrt{2}}{Z}-\frac{5}{2} \omega^{2} \tau_{n d}^{2}-\left(\frac{23}{4}+\frac{\sqrt{2}}{Z}\right) i \omega \tau_{n d}}
$$


TABLE I. The values of $Q$ for different elements: hydrogen $(Z=1)$; helium $(Z=2)$; carbon $(Z=6)$; oxygen $(Z=8)$; iron $(Z=26)$, which may be expected in the outer layers of white dwarfs and neutron stars.

\begin{tabular}{ccccccc}
\hline \hline $\mathbf{Z}$ & 1 & 2 & 6 & 8 & 26 & $\infty$ \\
\hline $\mathbf{Q}$ & 0.23 & 0.365 & 0.62 & 0.68 & 0.87 & 1 \\
\hline
\end{tabular}

$$
a_{1}=-\frac{5}{2} \tau_{n d} \frac{1-i \omega \tau_{n d}}{1+\frac{\sqrt{2}}{Z}-\frac{5}{2} \omega^{2} \tau_{n d}^{2}-\left(\frac{23}{4}+\frac{\sqrt{2}}{Z}\right) i \omega \tau_{n d}}
$$

$$
\begin{aligned}
& a_{0}^{1}=\frac{15}{4} \tau_{n d} \frac{1+\frac{\sqrt{2}}{Z}-\frac{5}{2} \omega^{2} \tau_{n d}^{2}}{\left(1+\frac{\sqrt{2}}{Z}\right)^{2}+\left(\frac{449}{16}+\frac{13}{2} \frac{\sqrt{2}}{Z}+\frac{2}{Z^{2}}\right) \omega^{2} \tau_{n d}^{2}+\frac{25}{4} \omega^{4} \tau_{n d}^{4}} \\
& b_{0}^{1}=\frac{15}{4} \frac{\omega \tau_{n d}^{2}}{B} \frac{\frac{23}{4}+\frac{\sqrt{2}}{Z}}{\left(1+\frac{\sqrt{2}}{Z}\right)^{2}+\left(\frac{449}{16}+\frac{13}{2} \frac{\sqrt{2}}{Z}+\frac{2}{Z^{2}}\right) \omega^{2} \tau_{n d}^{2}+\frac{25}{4} \omega^{4} \tau_{n d}^{4}}, \\
& a_{1}^{1}=-\frac{5}{2} \tau_{n d} \frac{1+\frac{\sqrt{2}}{Z}+\left(\frac{13}{4}+\frac{\sqrt{2}}{Z}\right) \omega^{2} \tau_{n d}^{2}}{\left(1+\frac{\sqrt{2}}{Z}\right)^{2}+\left(\frac{449}{16}+\frac{13}{2} \frac{\sqrt{2}}{Z}+\frac{2}{Z^{2}}\right) \omega^{2} \tau_{n d}^{2}+\frac{25}{4} \omega^{4} \tau_{n d}^{4}} \\
& b_{1}^{1}=-\frac{5}{2} \frac{\omega \tau_{n d}^{2}}{B} \frac{\frac{19}{4}+\frac{5}{2} \omega^{2} \tau_{n d}^{2}}{\left(1+\frac{\sqrt{2}}{Z}\right)^{2}+\left(\frac{449}{16}+\frac{13}{2} \frac{\sqrt{2}}{Z}+\frac{2}{Z^{2}}\right) \omega^{2} \tau_{n d}^{2}+\frac{25}{4} \omega^{4} \tau_{n d}^{4}},
\end{aligned}
$$

In 3-polynomial approximation the solution of the system (147) is written as

$$
\begin{aligned}
& a_{0}=\frac{165}{32} \tau_{n d} \frac{1+\frac{15 \sqrt{2}}{11 Z}-\frac{35}{11} i \omega \tau_{n d}}{1+\frac{61 \sqrt{2}}{16 Z}+\frac{9}{2 Z^{2}}-\left(\frac{5385}{128}+\frac{365 \sqrt{2}}{32 Z}\right) \omega^{2} \tau_{n d}^{2}-i \omega \tau_{n d}\left(\frac{1017}{64}+\frac{667 \sqrt{2}}{32 Z}+\frac{9}{2 Z^{2}}-\frac{175}{16} \omega^{2} \tau_{n d}^{2}\right)} \\
& a_{1}=-\frac{65}{8} \tau_{n d} \frac{1+\frac{45 \sqrt{2}}{52 Z}-\frac{35}{26} \omega^{2} \tau_{n d}^{2}-\left(\frac{713}{208}+\frac{45 \sqrt{2}}{52 Z}\right) i \omega \tau_{n d}}{1+\frac{61 \sqrt{2}}{16 Z}+\frac{9}{2 Z^{2}}-\left(\frac{5385}{128}+\frac{365 \sqrt{2}}{32 Z}\right) \omega^{2} \tau_{n d}^{2}-i \omega^{2} \tau_{n d}^{2}\left(\frac{1017}{64}+\frac{667 \sqrt{2}}{32 Z}+\frac{9}{2 Z^{2}}-\frac{175}{16} \omega^{2} \tau_{n d}^{2}\right)}
\end{aligned}
$$

$$
\begin{aligned}
& a_{0}^{1}=\frac{165}{32} \tau_{n d} \frac{\left(1+\frac{15 \sqrt{2}}{11 Z}\right)\left[1+\frac{61 \sqrt{2}}{16 Z}+\frac{9}{2 Z^{2}}-\left(\frac{5385}{128}+\frac{365 \sqrt{2}}{32 Z}\right) \omega^{2} \tau_{n d}^{2}\right]+\frac{35}{11} \omega^{2} \tau_{n d}^{2}\left(\frac{1017}{64}+\frac{667 \sqrt{2}}{32 Z}+\frac{9}{2 Z^{2}}-\frac{175}{16} \omega^{2} \tau_{n d}^{2}\right)}{\left[1+\frac{61 \sqrt{2}}{16 Z}+\frac{9}{2 Z^{2}}-\left(\frac{5385}{128}+\frac{365 \sqrt{2}}{32 Z}\right) \omega^{2} \tau_{n d}^{2}\right]^{2}+\omega^{2} \tau_{n d}^{2}\left(\frac{1017}{64}+\frac{667 \sqrt{2}}{32 Z}+\frac{9}{2 Z^{2}}-\frac{175}{16} \omega^{2} \tau_{n d}^{2}\right)^{2}} \\
& b_{0}^{1}=\frac{165}{32} \frac{\omega \tau_{n d}^{2}}{B} \frac{-\frac{35}{11}\left[1+\frac{61 \sqrt{2}}{16 Z}+\frac{9}{2 Z^{2}}-\left(\frac{5385}{128}+\frac{365 \sqrt{2}}{32 Z}\right) \omega^{2} \tau_{n d}^{2}\right]+\left(1+\frac{15 \sqrt{2}}{11 Z}\right)\left(\frac{1017}{64}+\frac{667 \sqrt{2}}{32 Z}+\frac{9}{2 Z^{2}}-\frac{175}{16} \omega^{2} \tau_{n d}^{2}\right)}{\left[1+\frac{61 \sqrt{2}}{16 Z}+\frac{9}{2 Z^{2}}-\left(\frac{5385}{128}+\frac{365 \sqrt{2}}{32 Z}\right) \omega^{2} \tau_{n d}^{2}\right]^{2}+\omega^{2} \tau_{n d}^{2}\left(\frac{1017}{64}+\frac{667 \sqrt{2}}{32 Z}+\frac{9}{2 Z^{2}}-\frac{175}{16} \omega^{2} \tau_{n d}^{2}\right)^{2}}
\end{aligned}
$$




$$
\begin{gathered}
a_{1}^{1}=-\frac{65}{8} \tau_{n d} \frac{\left(1+\frac{45 \sqrt{2}}{52 Z}-\frac{35}{26} \omega^{2} \tau_{n d}^{2}\right)\left[1+\frac{61 \sqrt{2}}{16 Z}+\frac{9}{2 Z^{2}}-\left(\frac{5385}{128}+\frac{365 \sqrt{2}}{32 Z}\right) \omega^{2} \tau_{n d}^{2}\right]+\left(\frac{713}{208}+\frac{45 \sqrt{2}}{52 Z}\right) \omega^{2} \tau_{n d}^{2}\left(\frac{1017}{64}+\frac{667 \sqrt{2}}{32 Z}+\frac{9}{2 Z^{2}}-\frac{175}{16} \omega^{2} \tau_{n d}^{2}\right)}{\left[1+\frac{61 \sqrt{2}}{16 Z}+\frac{9}{2 Z^{2}}-\left(\frac{5385}{128}+\frac{365 \sqrt{2}}{32 Z}\right) \omega^{2} \tau_{n d}^{2}\right]^{2}+\omega^{2} \tau_{n d}^{2}\left(\frac{1017}{64}+\frac{667 \sqrt{2}}{32 Z}+\frac{9}{2 Z^{2}}-\frac{175}{16} \omega^{2} \tau_{n d}^{2}\right)^{2}} \\
b_{1}^{1}=-\frac{65}{8} \frac{\omega \tau_{n d}^{2}}{B} \frac{\left(1+\frac{45 \sqrt{2}}{52 Z}-\frac{35}{26} \omega^{2} \tau_{n d}^{2}\right)\left(\frac{1017}{64}+\frac{667 \sqrt{2}}{32 Z}+\frac{9}{2 Z^{2}}-\frac{175}{16} \omega^{2} \tau_{n d}^{2}\right)-\left(\frac{713}{208}+\frac{45 \sqrt{2}}{52 Z}\right)\left[1+\frac{61 \sqrt{2}}{16 Z}+\frac{9}{2 Z^{2}}-\left(\frac{5385}{128}+\frac{365 \sqrt{2}}{32 Z}\right) \omega^{2} \tau_{n d}^{2}\right]}{\left[1+\frac{61 \sqrt{2}}{16 Z}+\frac{9}{2 Z^{2}}-\left(\frac{5385}{128}+\frac{365 \sqrt{2}}{32 Z}\right) \omega^{2} \tau_{n d}^{2}\right]^{2}+\omega^{2} \tau_{n d}^{2}\left(\frac{1017}{64}+\frac{667 \sqrt{2}}{32 Z}+\frac{9}{2 Z^{2}}-\frac{175}{16} \omega^{2} \tau_{n d}^{2}\right)^{2}}
\end{gathered}
$$

The values $c_{0}^{1}$ and $c_{1}^{1}$ in 2 and 3 polynomial approximations are defined using (107).
The heat flux $q_{i}$ from (105), (108) may be written in the form

$$
\begin{gathered}
q_{i}=-\frac{5}{2} \frac{k^{2} T n_{e}}{m_{e}}\left[\left(a_{0}^{1}-a_{1}^{1}\right) \delta_{i k}-\varepsilon_{i k n} B_{n}\left(b_{0}^{1}-b_{1}^{1}\right)+B_{i} B_{k}\left(c_{0}^{1}-c_{1}^{1}\right)\right] \frac{\partial T}{\partial r_{k}}=q_{i}^{(1)}+q_{I}^{(2)}+q_{i}^{(3)}, \\
q_{i}^{(1)}=-\frac{5}{2} \frac{k^{2} T n_{e}}{m_{e}}\left(a_{0}^{1}-a_{1}^{1}\right) \frac{\partial T}{\partial r_{i}}=-\lambda_{n d}^{(1)} \frac{\partial T}{\partial r_{i}} \\
q_{i}^{(2)}=\frac{5}{2} \frac{k^{2} T n_{e}}{m_{e}} \varepsilon_{i k n} B_{n}\left(b_{0}^{1}-b_{1}^{1}\right) \frac{\partial T}{\partial r_{k}}=-\varepsilon_{i k n} B_{n} \lambda_{n d}^{(2)} \frac{\partial T}{\partial r_{k}} \\
q_{i}^{(3)}=-\frac{5}{2} \frac{k^{2} T n_{e}}{m_{e}} B_{i} B_{k}\left(c_{0}^{1}-c_{1}^{1}\right) \frac{\partial T}{\partial r_{k}}=-B_{i} B_{k} \lambda_{n d}^{(3)} \frac{\partial T}{\partial r_{k}} .
\end{gathered}
$$

For 2-polynomial approximation we obtain

$$
\begin{gathered}
\lambda_{n d}^{(12)}=\frac{5}{2} \frac{k^{2} T n_{e}}{m_{e}}\left(a_{0}^{1}-a_{1}^{1}\right)=\frac{25}{4} \frac{k^{2} T n_{e}}{m_{e}} \tau_{n d} \frac{\frac{5}{2}\left(1+\frac{\sqrt{2}}{Z}\right)+\left(-\frac{1}{2}+\frac{\sqrt{2}}{Z}\right) \omega^{2} \tau_{n d}^{2}}{\left(1+\frac{\sqrt{2}}{Z}\right)^{2}+\left(\frac{449}{16}+\frac{13}{2} \frac{\sqrt{2}}{Z}+\frac{2}{Z^{2}}\right) \omega^{2} \tau_{n d}^{2}+\frac{25}{4} \omega^{4} \tau_{n d}^{4}}, \\
\lambda_{n d}^{(22)}=-\frac{5}{2} \frac{k^{2} T n_{e}}{m_{e}}\left(b_{0}^{1}-b_{1}^{1}\right)=-\frac{25}{4} \frac{k^{2} T n_{e}}{m_{e}} \frac{\omega \tau_{n d}^{2}}{B} \frac{\frac{107}{8}+\frac{3 \sqrt{2}}{2 Z}+\frac{5}{2} \omega^{2} \tau_{n d}^{2}}{\left(1+\frac{\sqrt{2}}{Z}\right)^{2}+\left(\frac{449}{16}+\frac{13}{2} \frac{\sqrt{2}}{Z}+\frac{2}{Z^{2}}\right) \omega^{2} \tau_{n d}^{2}+\frac{25}{4} \omega^{4} \tau_{n d}^{4}}, \\
B^{2} \lambda_{n d}^{(32)}=\lambda_{n d}^{(12)}(B=0)-\lambda_{n d}^{(12)} .
\end{gathered}
$$

The expressions for heat conductivity coefficients in 3 -polynomial approximation are very cumbersome, and are not presented here. They could be written explicitly using (123) - (130).

Using (108) we present another form of the components of the heat conductivity tensor in the magnetic field. Three components of the heat flux: parallel $q_{\|}$, perpendicular $q_{\perp}$ to the magnetic field $\vec{B}$, and "Hall" component of the heat flux $q_{\text {hall }}$, perpendicular to both vectors $\nabla T$ and $\vec{B}$, with account of (111) or (113) are defined by relations

$$
\begin{gathered}
q_{\|}=-\lambda_{\|} \nabla T_{\|}, \\
\lambda_{\|}=\frac{5}{2} \frac{k^{2} T n_{e}}{m_{e}}\left[a_{0}^{1}-a_{1}^{1}+B^{2}\left(c_{0}^{1}-c_{1}^{1}\right)\right]=\lambda_{n d}, \\
q_{\perp}=-\lambda_{\perp} \nabla T_{\perp}, \quad \lambda_{\perp}=\frac{5}{2} \frac{k^{2} T n_{e}}{m_{e}}\left(a_{0}^{1}-a_{1}^{1}\right), \\
q_{\text {hall }}=-\lambda_{\text {hall }} \frac{\nabla T \times \vec{B}}{B}, \quad \lambda_{\text {hall }}=\frac{5}{2} \frac{k^{2} T n_{e}}{m_{e}} B\left(b_{0}^{1}-b_{1}^{1}\right) .
\end{gathered}
$$




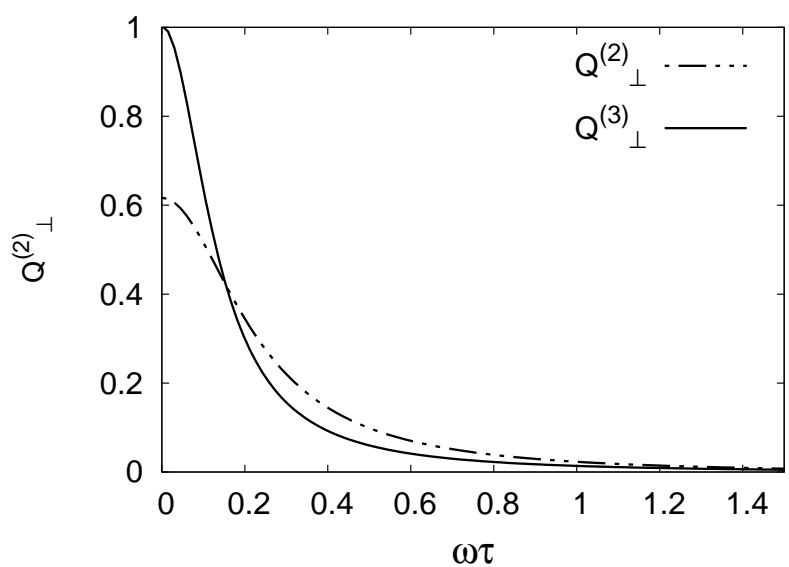

FIG. 1. Comparison of 2 and 3 polynomial approximation for nondegenerate carbon plasma at $\mathrm{Z}=6$, at different $\omega \tau$.

The 2-polynomial results coincide with corresponding derivations obtained in [23, 24].

The difference between 2 and 3 polynomial approximation may be characterised by comparison of values $Q_{\perp}^{(2)}$ and $Q_{\perp}^{(3)}$ in Fig.1.

$$
Q_{\perp}^{(2)}=\frac{\lambda_{n d}^{(12)}}{\lambda_{n d}^{(3)}}, \quad Q_{\perp}^{(3)}=\frac{\lambda_{n d}^{(13)}}{\lambda_{n d}^{(3)}},
$$

where $\lambda_{n d}^{(12)}$ is defined in (131), $\lambda_{n d}^{(3)}$ is defined in (113), and $\lambda_{n d}^{(13)}$ is defined from (128), (123), (125) in the same way as $\lambda_{n d}^{(12)}$. The functions $Q_{\perp}^{(2)}\left(\omega \tau_{n d}\right), Q_{\perp}^{(3)}\left(\omega \tau_{n d}\right)$ are presented in Fig. 1 for carbon, at $\mathrm{Z}=6$. In this figure we have $Q_{\perp}^{(2)}=0.023$ and $Q_{\perp}^{(3)}=0.014$, at $\omega \tau=1$.

\section{COMPARISON OF THE EXACT SOLUTION IN LORENTZ APPROXIMATION FOR A HEAT CONDUCTIVITY WITH POLYNOMIAL EXPANSION}

\section{A. Exact solution in Lorentz approximation}

The Lorentz approximation for solving a kinetic equation is applied when the mass of light particles (electrons) is much smaller than the the mass of heavy particles (nuclei), and in addition electron-electron collisions are neglected. In this approximation the linearized Boltzmann equation has an exact solution. This approximation works good for metal transport coefficients, where a strong electron degeneracy permits to neglect electron-electron collisions. Lorenz approximation is useful for checking approximate polynomial solution, because it gives a possibility to follow a convergence of the approximate solution to the exact one, increasing the number of polynomials. In different approaches the solution in Lorentz approximation was considered in [12], [39], [28], [40], , see also [35].

The explicit exact solution in Lorentz approximation is obtained for the case $B=0$. If we consider only the heat flux connected with the temperature gradient, at zero value of the diffusive vector $d_{i}$ from (19), (20), than we obtain the expression for the heat flux from [35] as

$$
q_{i}=-\frac{640 k}{\Lambda} \frac{m_{e}(k T)^{4}}{n_{N} Z^{2} e^{4} h^{3}}\left(G_{5}-\frac{1}{2} \frac{G_{5 / 2}}{G_{3 / 2}} G_{4}\right) \frac{\partial T}{\partial r_{\mathrm{i}}} .
$$

In the limiting cases the coefficient in (138) is reduces to

$$
\begin{aligned}
\lambda_{e}^{l}= & \frac{40 \sqrt{2}}{\pi^{3 / 2} \Lambda} k \frac{n_{e}}{n_{N}} \frac{(k T)^{5 / 2}}{\mathrm{e}^{4} Z^{2} \sqrt{m_{e}}}=\frac{320}{3 \pi} \frac{k^{2} T n_{e}}{m_{e}} \tau_{n d} \\
& =\frac{5}{64 \Lambda} \frac{k^{2} T n_{\mathrm{e}}^{2} h^{3}}{m_{\mathrm{e}}^{2} n_{N} Z^{2} e^{4}}=\frac{5 \pi^{2}}{6} \frac{k^{2} T n_{e}}{m_{e}} \tau_{d} \quad(D) .
\end{aligned}
$$

Note, that the heat conductivity coefficient in (139) determines the heat flux at zero value of the diffusion vector $d_{i}=0$. Often the heat conductivity coefficient is written for the case of zero value of the diffusion velocity $\left\langle v_{\alpha i}\right\rangle=0[12,35]$. When the thermal conductivity and diffusion are calculated in the same procedure, both heat and diffusion fluxes are calculated without any restrictions on the diffusion vector or diffusion velocity. Such consideration will be performed elsewhere. The exact formulae in the Lorentz model are used [12] for estimation of the precision of the polynomial approximation.

The input of electron-electron collisions into the heat conductivity coefficients for different $Z$ may be estimated from the plot of normalized 3-polynomial heat conductivity coefficients in the direction perpendicular to the magnetic field, introducing the value $Q_{\perp}^{(3 l)}$, defined as

$$
Q_{\perp}^{(3 l)}=\frac{\lambda_{n d}^{(13)}}{\lambda_{e, n d}^{l}} .
$$

Here $\lambda_{e, n d}^{l}$ is taken from the upper line in (139). The curves of this value, for different $Z$, including $Z=\infty$, related to Lorentz approximation, are plotted in Fig.2. The intersection of the plots with the y-axis in Fig.2 occurs in the points given in the Table 1, multiplied by $\frac{\lambda_{n d}^{(3)}}{\lambda_{e, n d}^{l}}=0,978$. At $\omega \tau=1$ we have $Q_{\perp}^{(3 l)}=$ $0.0053,0.0060,0.0083,0.014$ for $Z=\infty, 26,6,2$ respectively.

\section{B. Polynomial calculations without account of collisions between electrons}

In order to test the precision of polynomial approximation for the heat conductivity coefficients we compare 


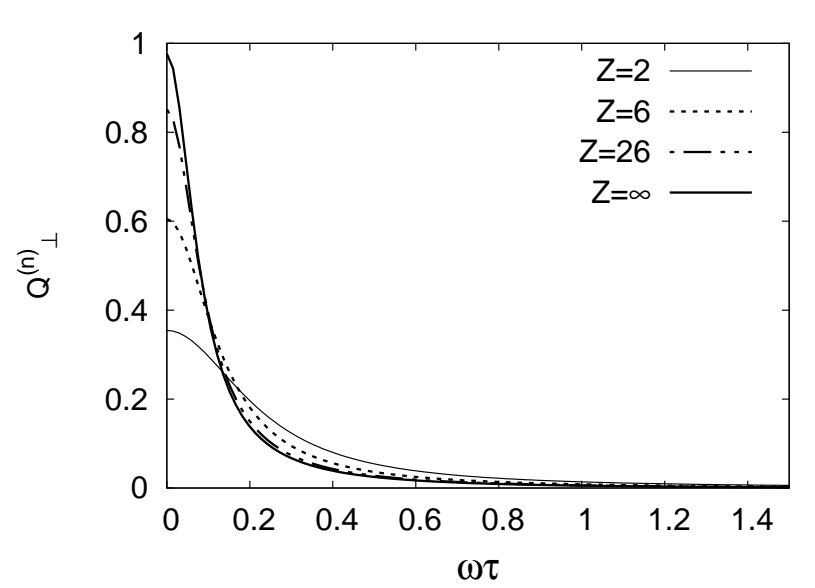

them with ones, obtained by exact solution in Lorentz approximation. Omitting electron-electron collisions we obtain in 3 polynomial approximation the following system

FIG. 2. The plots of the value $Q_{\perp}^{(3 l)}$ as a function of $\omega \tau$ in 3 polynomial approximation are presented for nondegenerate plasma of helium $(Z=2)$, carbon $(Z=6)$, iron $(Z=26)$, for comparison with the Lorentz plasma, formally corresponding to $Z=\infty$. The deviations from the Lorentz plasma are connected with the input of electron-electron collisions. The intersection of Lorentz 3-polynomial curve $(\mathrm{Z}=\infty)$ with $\mathrm{y}$-axis at 0.978 is connected with deviation from the exact solution in Lorentz approximation.

$$
\left\{\begin{array}{r}
0=-\frac{3}{2} i \omega n_{e} a_{0}+a_{0} b_{00}+a_{1} b_{01}+a_{2} b_{02} \\
-\frac{15}{4} n_{e}\left(\frac{7 G_{7 / 2}}{2 G_{3 / 2}}-\frac{5 G_{5 / 2}^{2}}{2 G_{3 / 2}^{2}}\right)=-\frac{15}{4}\left(\frac{7 G_{7 / 2}}{2 G_{3 / 2}}-\frac{5 G_{5 / 2}^{2}}{2 G_{3 / 2}^{2}}\right) i \omega n_{e} a_{1}+a_{0} b_{10}+a_{1} b_{11}+a_{2} b_{12} \\
0=-\frac{105}{16}\left(-\frac{35}{8} \frac{G_{7 / 2}^{2}}{G_{3 / 2}^{2}}+\frac{49}{2} \frac{G_{7 / 2}^{2}}{G_{5 / 2}^{2}} \frac{G_{7 / 2}}{G_{3 / 2}}-\frac{63}{2} \frac{G_{9 / 2} G_{7 / 2}}{G_{5 / 2} G_{3 / 2}}+\frac{99}{8} \frac{G_{11 / 2}}{G_{3 / 2}}\right) i \omega n_{e} a_{2}+a_{0} b_{20}+a_{1} b_{21}+a_{2} b_{22}
\end{array}\right.
$$

\section{Results for non-degenerate electrons}

In absence of the magnetic field, in Lorenz approximation with $a_{j k}=0$, the system (32) is reduced to

$$
\left\{\begin{aligned}
0 & =a_{0} b_{00}+a_{1} b_{01}+a_{2} b_{02} \\
-\frac{15}{4} n_{e} & =a_{0} b_{10}+a_{1} b_{11}+a_{2} b_{12} \\
0 & =a_{0} b_{20}+a_{1} b_{21}+a_{2} b_{22}
\end{aligned}\right.
$$

With account of (62)- (67) this system is written as

$$
\left\{\begin{array}{r}
0=\frac{3}{2} a_{0}+\frac{9}{4} a_{1}+\frac{45}{16} a_{2} \\
-\frac{15}{4} \tau_{n d}=\frac{9}{4} a_{0}+\frac{39}{8} a_{1}+\frac{207}{32} a_{2} \\
0=\frac{45}{16} a_{0}+\frac{207}{32} a_{1}+\frac{1299}{128} a_{2}
\end{array}\right.
$$

This system is written for 3-polynomial approximation to the solution. Two first equations at $a_{2}=0$ determine the 2-polynomial approximation, giving with account of (108) the following results

$$
\begin{gathered}
a_{0}=\frac{15}{4} \tau_{n d}, \quad a_{1}=-\frac{5}{2} \tau_{n d}, \\
\lambda_{n d l}^{(2)}=\frac{25}{4} \frac{5}{2} \frac{k^{2} T n_{e}}{m_{e}} \tau_{n d}=15.63 \frac{k^{2} T n_{e}}{m_{e}} \tau_{n d} .
\end{gathered}
$$

In 3-polynomial approximation we obtain the solution of (143) for $a_{0}, a_{1}$, and heat conductivity coefficient, with account of (108), as

$$
\begin{gathered}
a_{0}=\frac{165}{32} \tau_{n d}, \quad a_{1}=-\frac{65}{8} \tau_{n d}, \\
\lambda_{n d l}^{(3)}=\frac{425}{32} \frac{5}{2} \frac{k^{2} T n_{e}}{m_{e}} \tau_{n d}
\end{gathered}
$$




$$
=\frac{2125}{64} \frac{k^{2} T n_{e}}{m_{e}} \tau_{n d}=33.20 \frac{k^{2} T n_{e}}{m_{e}} \tau_{n d} .
$$

The heat coefficients obtained by the method of successive polynomial approximations should be compared with the exact solution $\lambda_{n d}^{l}$, obtained by Lorentz method (139) for non-degenerate electrons

$$
\lambda_{n d}^{(l)}=\frac{320}{3 \pi} \frac{k^{2} T n_{e}}{m_{e}} \tau_{n d}=33.95 \frac{k^{2} T n_{e}}{m_{e}} \tau_{n d} .
$$

It is clear that the 2- polynomial solution underestimate the coefficient of the heat conductivity for more than $50 \%$, and the 3 -polynomial solution differs from the exact solution only for about $2.2 \%$. Equations or 3 polynomial approximation in presence of a magnetic field are obtained from (141) with account of (62)-(67), (143) in the form

$$
\left\{\begin{array}{r}
0=-\frac{3}{2} i \omega \tau_{n d} a_{0}+\frac{3}{2} a_{0}+\frac{9}{4} a_{1}+\frac{45}{16} a_{2} \\
-\frac{15}{4} \tau_{n d}=-\frac{15}{4} i \omega \tau_{n d} a_{1}+\frac{9}{4} a_{0}+\frac{39}{8} a_{1}+\frac{207}{32} a_{2} \\
0=-\frac{105}{16} i \omega \tau_{n d} a_{2}+\frac{45}{16} a_{0}+\frac{207}{32} a_{1}+\frac{1299}{128} a_{2}
\end{array}\right.
$$

Explicit solution of equations (147) for 2 and 3 polynomial approximations is determined by formulae (115)(126) at formally infinite value of $Z$.

\section{Partially degenerate electrons}

For partially degenerate electrons at $x_{0}=0$, with the degeneracy level $D L=\frac{\varepsilon_{f e}}{k T}=1.011$, the system (141) is written in the form

$$
\left\{\begin{array}{c}
0=-1.5 i \omega n_{e} a_{0}+a_{0} b_{00}+a_{1} b_{01}+a_{2} b_{02} \\
-3.88 n_{e}=-3.88 i \omega n_{e} a_{1}+a_{0} b_{10}+a_{1} b_{11}+a_{2} b_{12} \\
0=-7.138 i \omega n_{e} a_{2}+a_{0} b_{20}+a_{1} b_{21}+a_{2} b_{22}
\end{array}\right.
$$

where matrix elements $b_{j k}$ are defined in (76)-(78). In absence of a magnetic field this system is reduced to

$$
\left\{\begin{aligned}
0 & =a_{0} b_{00}+a_{1} b_{01}+a_{2} b_{02} \\
-3.88 n_{e} & =a_{0} b_{10}+a_{1} b_{11}+a_{2} b_{12} \\
0 & =a_{0} b_{20}+a_{1} b_{21}+a_{2} b_{22}
\end{aligned}\right.
$$

With account of (76)-(178) this system may be written in the form

$$
\left\{\begin{array}{r}
0=1.5 a_{0}+2.16 a_{1}+2.588 a_{2} \\
-3.88 \tau_{d 0}=2.16 a_{0}+5.162 a_{1}+6.671 a_{2} \\
0=2.588 a_{0}+6.671 a_{1}+11.038 a_{2}
\end{array}\right.
$$

This system is written for 3-polynomial approximation to the solution. Two first equations at $a_{2}=0$ determine the 2-polynomial approximation, which with account of (106), give the following result

$$
\begin{gathered}
a_{0}=2.723 \tau_{d 0}, \quad a_{1}=-1.891 \tau_{d 0}, \\
\lambda_{d 0 l}^{(2)}=5.043 \frac{5}{2} \frac{k^{2} T n_{e}}{m_{e}} \tau_{n d}=12.61 \frac{k^{2} T n_{e}}{m_{e}} \tau_{d 0} .
\end{gathered}
$$

In 3-polynomial approximation we obtain the solution of (150) for $a_{0}, a_{1}$, and heat conductivity coefficient, with account of (106), as

$$
\begin{gathered}
a_{0}=3.533 \tau_{d 0}, \quad a_{1}=-5.295 \tau_{d 0}, \\
\lambda_{d 0 l}^{(3)}=8.278 \frac{5}{2} \frac{k^{2} T n_{e}}{m_{e}} \tau_{d 0}=22.07 \frac{k^{2} T n_{e}}{m_{e}} \tau_{d 0} .
\end{gathered}
$$

The heat coefficients obtained by the method of successive polynomial approximations should be compared with an exact solution $\lambda_{n d}^{l}$ obtained by Lorentz method (138) for non-degenerate electrons

$$
\lambda_{d 0}^{(l)}=0.744 \frac{320}{3 \pi} \frac{k^{2} T n_{e}}{m_{e}} \tau_{d 0}=25.26 \frac{k^{2} T n_{e}}{m_{e}} \tau_{d 0} .
$$

It is clear that the 2- polynomial solution underestimate the coefficient of the heat conductivity for more than $50 \%$, and the 3-polynomial solution differs from the exact solution for about $13 \%$. So, a convergence of the polynomial approximation to the exact value takes place slower than for non-degenerate electrons in previous subsection.

\section{E. Results for strongly degenerate electrons}

The non-diagonal matrix elements $b_{i k}, i \neq k$ for strongly degenerate case are much smaller than the diagonal one $b_{i i}$, according to (88)- (90). In this case at $x_{0} \gg 1$, and neglecting terms $\sim 1 / x_{0}$ we obtain a simplified system (141) for 3-polynomial expansion as 


$$
\left\{\begin{array}{c}
0=-\frac{3}{2} i \omega n_{e} a_{0}+a_{0} b_{00} \\
-\frac{15}{4} n_{e} \frac{2 \pi^{2}}{15}=-\frac{15}{4} \frac{2 \pi^{2}}{15} i \omega n_{e} a_{1}+a_{1} b_{11} \\
0=-\frac{105}{16}\left(-\frac{35}{8} \frac{G_{7 / 2}^{2}}{G_{3 / 2}^{2}}+\frac{49}{2} \frac{G_{7 / 2}^{2}}{G_{5 / 2}^{2}} \frac{G_{7 / 2}}{G_{3 / 2}}-\frac{63}{2} \frac{G_{9 / 2} G_{7 / 2}}{g_{5 / 2} G_{3 / 2}}+\frac{99}{8} \frac{G_{11 / 2}}{G_{3 / 2}}\right) i \omega n_{e} a_{2}+a_{2} b_{22}
\end{array}\right.
$$

Solution of the system (154) is written in the form, with account of (80), (89)

$$
a_{0}=0, \quad a_{2}=0,
$$

$$
a_{1}=\frac{\frac{15}{4} n_{e} \frac{2 \pi^{2}}{15}}{\frac{15}{4} \frac{2 \pi^{2}}{15} i n_{e} \omega-b_{11}}=\frac{\frac{\pi^{2} n_{e}}{2}}{i \omega \frac{\pi^{2} n_{e}}{2}-\frac{\pi^{2} n_{e}}{2 \tau_{d}}}=-\frac{\tau_{d}}{1-i \omega \tau_{d}}=-\tau_{d} \frac{1+i \omega \tau_{d}}{1+\omega^{2} \tau_{d}^{2}}=a_{1}^{1}+i B b_{1}^{1},
$$

$$
c_{1}^{1}=-\frac{\tau_{d}}{B^{2}} \frac{\omega^{2} \tau_{d}^{2}}{1+\omega^{2} \tau_{d}^{2}} .
$$

Using (106) we obtain the components of the heat conductivity tensor in the magnetic field for strongly degenerate electrons in polynomial approximation. 3 components of the heat flux: parallel $q_{\|}^{s d}$, perpendicular $q_{\perp}^{(s d)}$ to the magnetic field $\vec{B}$, and "Hall" component of the heat flux $q_{\text {hall }}^{(s d)}$, perpendicular to both vectors $\nabla T$ and $\vec{B}$, with account of (138),(155)-(156) are defined by relations

$$
\begin{gathered}
q_{\|}^{(s d)}=-\lambda_{\|}^{(s d)} \nabla T_{\|}, \\
\lambda_{\|}^{(s d)}=-\frac{\pi^{2}}{3} \frac{k^{2} T n_{e}}{m_{e}}\left(a_{1}^{1}+B^{2} c_{1}^{1}\right)=\frac{\pi^{2}}{3} \frac{k^{2} T n_{e}}{m_{e}} \tau_{d} . \\
q_{\perp}^{(s d)}=-\lambda_{\perp}^{(2)} \nabla T_{\perp}, \quad \lambda_{\perp}^{(s d)}=-\frac{\pi^{2}}{3} \frac{k^{2} T n_{e}}{m_{e}} a_{1}^{1} \\
=\frac{\pi^{2}}{3} \frac{k^{2} T n_{e}}{m_{e}} \frac{\tau_{d}}{1+\omega^{2} \tau_{d}^{2}} \\
q_{\text {hall }}^{(s d)}=-\lambda_{\text {hall }}^{(2)} \frac{\nabla T \times B}{B}, \quad \lambda_{\text {hall }}^{(s d)}=-\frac{\pi^{2}}{3} \frac{k^{2} T n_{e}}{m_{e}} B b_{1}^{1} .
\end{gathered}
$$

Comparing (160) with the exact value for strongly degenerate electrons from Lorentz approximation (139) we see, that polynomial approximation, where the terms $\sim x_{0}^{-1}$ are neglected, gives is 2.5 times smaller value than the exact one. This value, as well as a simple dependence of the heat conductivity tensor on the magnetic field $q_{\|}^{(s d)} / q_{\perp}^{(s d)}=\left(1+\omega^{2} \tau_{d}^{2}\right)$ follows also from a rough theory of heat conductivity and diffusion in presence of a magnetic field, based on the mean free path, which is described in [12]. The value of the heat conductivity coefficient, following from this approach, was considered in [8],[9], and many subsequent papers. As mentioned above the heat flux calculated here is connected only with a temperature gradient, when the diffusion vector $d_{i}=0$. In laboratory conditions when the electrical conductivity is small and electrical current is damped rapidly, another limiting case is considered, where $j_{i} \sim\left\langle v_{i}\right\rangle=0$. This restriction leads to linear connection between $d_{i}$ and $\nabla T$, what permits [12] to exclude $d_{i}$ and to express the heat flux as directly proportional to $\nabla T$, with another heat conductivity coefficient $\lambda_{j}$. For strongly degenerate electrons we have $\lambda_{j}=0.4 \lambda_{e}^{l}=\lambda_{\|}^{(s d)}$, see (139), (158) and [35].

\section{HEAT CONDUCTIVITY OF STRONGLY DEGENERATE ELECTRONS IN PRESENCE OF MAGNETIC FIELD: LORENTZ APPROXIMATION}

The equation for the function $\xi$ from (19), (23) may be written in the form, using relations $f_{0}^{\prime}=f_{0}, \xi^{\prime}=\xi, u_{i}^{\prime}=$ $u_{i} \cos \theta$, and making integration over $d c_{N i}$ with account of (14), as 


$$
f_{0}\left(1-f_{0}\right)\left(u^{2}-\frac{5 G_{5 / 2}}{2 G_{3 / 2}}\right)=-i B f_{0}\left(1-f_{0}\right) \frac{e \xi}{m_{e} c} u_{i}+f_{0}\left(1-f_{0}\right) n_{N} \xi \int(1-\cos \theta) g_{e N} b d b d \varepsilon
$$

The function $\xi$ is defined by expression

$$
\xi=\frac{u^{2}-\frac{5}{2} \frac{G_{5 / 2}}{G_{3 / 2}}}{2 \pi n_{N} \int_{0}^{\infty}(1-\cos \theta) g b d b-i \omega}
$$

Using (46)-(50) we obtain in Lorenz approximation, with $g_{12}=v$,

$$
\begin{gathered}
\int_{0}^{\infty}(1-\cos \theta) g b d b=2 \frac{e^{4} Z^{2}}{m_{e}^{2} v^{3}} \Lambda \\
\xi=\frac{u^{2}-\frac{5}{2} \frac{G_{5 / 2}}{G_{3 / 2}}}{4 \pi n_{N}\left(\frac{m_{e}}{2 k T}\right)^{3 / 2} \frac{e^{4} Z^{2}}{m_{e}^{2} u^{3}} \Lambda-i \omega} .
\end{gathered}
$$

According to (22) we have

$$
\begin{gathered}
q_{i}=-\frac{2 \pi}{3} \frac{m_{e}^{4}}{h^{3} T}\left(\frac{2 k T}{m_{e}}\right)^{7 / 2}\left[\delta_{i j} \int_{0}^{\infty} f_{0}\left(1-f_{0}\right) A^{(1)} x^{5 / 2} d x-\varepsilon_{i j k} B_{k} \int_{0}^{\infty} f_{0}\left(1-f_{0}\right) A^{(2)} x^{5 / 2} d x\right. \\
\left.+B_{i} B_{j} \int_{0}^{\infty} f_{0}\left(1-f_{0}\right) A^{(3)} x^{5 / 2} d x\right] \frac{\partial T}{\partial x_{j}}=q_{i}^{(1)}+q_{i}^{(2)}+q_{i}^{(3)}, \quad x=u^{2}, \\
q_{i}^{(1)}=-\frac{2 \pi}{3} \frac{m_{e}^{4}}{h^{3} T}\left(\frac{2 k T}{m_{e}}\right)^{7 / 2} \int_{0}^{\infty} f_{0}\left(1-f_{0}\right) A^{(1)} x^{5 / 2} d x \frac{\partial T}{\partial x_{i}}=-\lambda_{s d}^{(1)} \frac{\partial T}{\partial x_{i}}, \\
q_{i}^{(2)}=\varepsilon_{i j k} B_{k} \frac{2 \pi}{3} \frac{m_{e}^{4}}{h^{3} T}\left(\frac{2 k T}{m_{e}}\right)^{7 / 2} \int_{0}^{\infty} f_{0}\left(1-f_{0}\right) A^{(2)} x^{5 / 2} d x \frac{\partial T}{\partial x_{j}}=-\varepsilon_{i j k} B_{k} \lambda_{s d}^{(2)} \frac{\partial T}{\partial x_{j}}, \\
q_{i}^{(3)}=-B_{i} B_{j} \frac{2 \pi}{3} \frac{m_{e}^{4}}{h^{3} T}\left(\frac{2 k T}{m_{e}}\right)^{7 / 2} \int_{0}^{\infty} f_{0}\left(1-f_{0}\right) A^{(3)} x^{5 / 2} d x \frac{\partial T}{\partial x_{j}}=-B_{i} B_{j} \lambda_{s d}^{(3)} \frac{\partial T}{\partial x_{j}},
\end{gathered}
$$

The expression for the heat flux, following from (11), (17), (19), (22), (164)-(168) is written as

$$
\begin{gathered}
A^{(1)}=\frac{\left(u^{2}-\frac{5}{2} \frac{G_{5 / 2}}{G_{3 / 2}}\right) 4 \pi n_{N}\left(\frac{m_{e}}{2 k T}\right)^{3 / 2} \frac{e^{4} Z^{2}}{m_{e}^{2} u^{3}} \Lambda}{\left[4 \pi n_{N}\left(\frac{m_{e}}{2 k T}\right)^{3 / 2} \frac{e^{4} Z^{2}}{m_{e}^{2} u^{3}} \Lambda\right]^{2}+\omega^{2}} . \\
A^{(2)}=\frac{\omega}{B} \frac{u^{2}-\frac{5}{2} \frac{G_{5 / 2}}{G_{3 / 2}}}{\left[4 \pi n_{N}\left(\frac{m_{e}}{2 k T}\right)^{3 / 2} \frac{e^{4} Z^{2}}{m_{e}^{2} u^{3}} \Lambda\right]^{2}+\omega^{2}} . \\
A^{(3)}=A^{(1)}(B=0)-A^{(1)} .
\end{gathered}
$$

For strongly degenerate electrons at $x_{0} \gg 1$ the integrals in (169) with $A^{1}, A^{2} A^{(3)}$ from (166)-(168), are expressed analytically , using expansion formula [32])

$$
\int_{0}^{\infty} \frac{f(x) d x}{e^{x-x_{0}}+1}=\int_{0}^{x_{0}} f(x) d x+\frac{\pi^{2}}{6} f^{\prime}\left(x_{0}\right)+\ldots
$$

After partial integration we obtain the expression, which are suitable for integration by formula (170)

$$
\lambda^{(1)}=\frac{2 \pi}{3} \frac{m_{e}^{4}}{h^{3} T}\left(\frac{2 k T}{m_{e}}\right)^{7 / 2} \int_{0}^{\infty} f_{0} \frac{d\left(A^{(1)} x^{5 / 2}\right)}{d x} d x
$$

$$
\lambda^{(2)}=-\frac{2 \pi}{3} \frac{m_{e}^{4}}{h^{3} T}\left(\frac{2 k T}{m_{e}}\right)^{7 / 2} \int_{0}^{\infty} f_{0} \frac{d\left(A^{(2)} x^{5 / 2}\right)}{d x} d x
$$

$$
\begin{gathered}
B^{2} A^{(3)}=A^{(1)}(B=0)-A^{(1)}, \\
B^{2} \lambda^{(3)}=\lambda^{(1)}(B=0)-\lambda^{(1)} .
\end{gathered}
$$

Applying (170) to the integrals (171), (172), we obtain 


$$
\begin{aligned}
& \lambda^{(1)}=\frac{2 \pi}{3} \frac{m_{e}^{4}}{h^{3} T}\left(\frac{2 k T}{m_{e}}\right)^{7 / 2}\left[A^{(1)}\left(x_{0}\right) x_{0}^{5 / 2}+\left.\frac{\pi^{2}}{6} \frac{d^{2}\left(A^{(1)} x^{5 / 2}\right)}{d x^{2}}\right|_{x=x_{0}}\right], \\
& \lambda^{(2)}=-\frac{2 \pi}{3} \frac{m_{e}^{4}}{h^{3} T}\left(\frac{2 k T}{m_{e}}\right)^{7 / 2}\left[A^{(2)}\left(x_{0}\right) x_{0}^{5 / 2}+\left.\frac{\pi^{2}}{6} \frac{d^{2}\left(A^{(2)} x^{5 / 2}\right)}{d x^{2}}\right|_{x=x_{0}}\right],
\end{aligned}
$$

Using (166), (167), and writing the formula using $\tau_{d}$ from (57, we write the heat conductivity coefficients in the form

$$
\begin{gathered}
\lambda^{(1)}=\frac{5 \pi^{2}}{6} \frac{k^{2} T n_{e}}{m_{e}} \tau_{d}\left\{\frac{1}{1+\omega^{2} \tau_{d}^{2}}-\frac{6}{5} \frac{\omega^{2} \tau_{d}^{2}}{\left(1+\omega^{2} \tau_{d}^{2}\right)^{2}}-\left.\frac{\pi^{2}}{10}\left[\frac{1}{1+\omega^{2} \tau_{d}^{2}\left(\frac{x^{3}}{x_{0}^{3}}\right)}\right]^{\prime \prime}\right|_{x=x_{0}}\right\}, \\
\lambda^{(2)}=-\frac{4 \pi^{2}}{3} \frac{k^{2} T n_{e}}{m_{e}} \frac{\tau_{d}^{2} \omega}{B}\left\{\frac{1}{1+\omega^{2} \tau_{d}^{2}}-\frac{3}{4} \frac{\omega^{2} \tau_{d}^{2}}{\left(1+\omega^{2} \tau_{d}^{2}\right)^{2}}-\left.\frac{\pi^{2}}{16}\left[\frac{1}{1+\omega^{2} \tau_{d}^{2}\left(\frac{x^{3}}{x_{0}^{3}}\right)}\right]^{\prime \prime}\right|_{x=x_{0}}\right\},
\end{gathered}
$$

In the case of strongly degenerate electrons the equations (166)-(169), (176, (177) give an asymptotically exact solution for the heat conductivity coefficients, because collisions between electrons can be neglected in this case. The difference between the exact $\left[\lambda^{(1)}\right] /\left[\lambda^{(1)}(B=0)\right]$ from (176), and phenomenological (1) account of the magnetic field influence on the heat conductivity coefficients is presented in Fig.3. Here the ratios between the values, which are perpendicular and parallel to magnetic field, are plotted for $k T=0.09 E_{f}$. At $\omega \tau=1.5$ the exact value of this ratio is 4 times smaller than the phenomenological one.

The heat flux defined in (169)-(177) corresponds to the situation when the diffusion vector $d_{1}$ from (20) is zero. In general case the heat and diffusion (electrical current) fluxes are connected with each other by diffusion vector $d_{i}$ and temperature gradient $\partial T / \partial x_{i}$ [12].

\section{DISCUSSION}

In this paper a thermal conductivity tensor is found for arbitrary degenerate non-relativistic electrons in presence of a non-quantizing magnetic field. For nondegenerate electrons the conductivity tensor is derived from the solution of a Boltzmann kinetic equation by classical Chapman - Enskog method using an expansion on the Sonyne polynomials, with remaining two and three terms. The electron-nucleus and electron-electron collisions are taken into consideration. The tensor of the thermal conductivity is written for arbitrary local direction of the magnetic field and the temperature gradient, in the Cartesian coordinate system, following 25]. Our results exactly coincide with the results of previous authors $[22-$ 24] in two polynomial case. The analytic solution in three polynomial approximation was not obtained before. The value of the thermal conductivity coefficient obtained in the well-known work of Braginskii [26] in two-polynomial

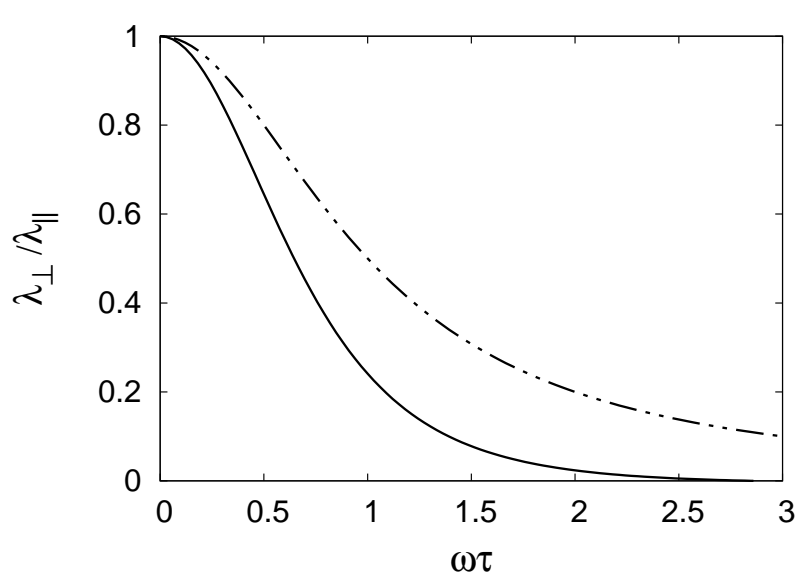

FIG. 3. The plots of the ratio $\lambda_{\perp} / \lambda_{\|}$as a function of $\omega \tau$ are presented for phenomenologically obtained heat conductivity (dash-dot line) for comparison with heat conductivity obtained by the solution of Boltzmann equation in Lorentz appoximation (solid line) with $k T=0.09 E_{f}$.

approximation is two times less than our correspondong value. It is connected with an approach used in the paper [26], which is different from the classical Chapman - Enskog method [12]. In his consideration one half of the thermal flux is hidden inside the so-called "thermal force", so that the resulting heat flux in the co-moving frame is the same in both considerations. The heat conductivity coefficients for strongly degenerate electrons, in presence of magnetic field, are obtained asymptotically exactly in Lorentz approximation, when the electronelectron collision may be neglected in comparison with electron-nuclei collisions at nondegenerate nuclei.

In most works considering the heat conductivity in astrophysical objects, in the neutron stars in particular, following Flowers and Itoh [8], the influence of the mag- 
netic field on the heat flux was taken into account phenomenologically using the coefficient $1 /\left(1+\omega^{2} \tau^{2}\right)$, which decreases the heat flux in the direction perpendicular to the direction of a magnetic field. Our results, obtained by the solution of Boltzmann equation show, that the influence of the magnetic field on the coefficients of heat conductivity is stronger, and has a more complicated character Fig.3.

On the example of the Lorentz approximation it was shown that the precision of approximation by the raw of the orthogonal functions, analogous to Sonyne polynomials, decreases with increasing of the degeneracy level. For strongly degenerate electrons the number of functions, needed for good precision is increasing $\sim x_{0}$, at $x_{0} \gg 1$, and for small number of number of functions the resulting heat conductivity coefficient at $B=0$ is 2.5 times smaller than the exact value. For moderately degenerate electrons with $x_{0}=0$ the approximation by three orthogonal functions gives the value of the heat conductivity coefficient approximately $13 \%$ smaller than the exact value (in Lorentz approximation, at $B=0$ ). In the same approximation for nondegenerate electrons the value of the heat conductivity coefficient is only $2.2 \%$ smaller than the exact value. Note that the electron-electron collision even more decrease the the value of the heat conductivity coefficients. Therefore, in three functional approximation, the Lorentz approach may give a more exact value for heat conductivity coefficient of moderately degenerate electrons, than with account of electron-electron collisions. The simple linear interpolation, between "exact" results for nondegenerate, and strongly degenerate electrons, may be suggested for all heat conductivity coefficients $\lambda_{i}\left(x_{0}\right)$ in presence of the magnetic field as

$$
\begin{aligned}
& \lambda_{i}\left(x_{0}\right)=\lambda_{i}^{(n d)} \frac{1-x_{0}}{2-x_{0}}+\lambda_{i}^{(s d)} \frac{1}{2-x_{0}}, \quad \text { at } \quad x_{0} \leq 0 \\
& \lambda_{i}\left(x_{0}\right)=\lambda_{i}^{(n d)} \frac{1}{2+x_{0}}+\lambda_{i}^{(s d)} \frac{1+x_{0}}{2+x_{0}}, \quad \text { at } \quad x_{0} \geq 0 .
\end{aligned}
$$

Here the indices (nd), (sd) are related to nondegenerate and strongly degenerate values, respectively.

The Chapmen-Enskog method could be used for sufficiently dense gas (plasma), where the time between collisions of particles is the smallest among other character- istic times. In presence of a magnetic field we have, in addition to the time of the system flyover, and characteristic time of the parameter variations in plasma, the time of the flight over Larmor circle $\tau_{L}=\frac{2 \pi}{\omega}$. This time should be much less than $\tau$, equal to $\tau_{n d}$ or $\tau_{d}$, what leads to inequality, at which the Chapmen-Enskog method could be used, in the form

$$
\omega \tau \ll 2 \pi
$$

Therefore the consideration in this paper could be safely applied at $\omega \tau \lesssim 1$, and for larger $\omega \tau$ only qualitative estimations could be obtained.

Our calculations have been done for non-relativistic electrons, while in deep layers of the neutron star crust the relativistic effects become important. The main relativistic effect of increasing the effective electron mass may be taken into account approximately, following [9], by writing in all expressions the relativistic electron mass $m_{e *}=\left(m_{e}^{2}+p_{F e}^{2} / c^{2}\right)^{1 / 2}$ instead of the rest mass $m_{e}$. The account of quantum effects is connected with consideration of discrete Landau levels in strong magnetic fields. This complicated problem is not yet solved.

The transport coefficients calculated here determine a heat flux carried by electrons in the case of zero diffusion vector $d_{i}$. In a general case of nonzero diffusion vector $d_{i}$ and temperature gradient $\partial T / \partial x_{i}$, the heat and diffusion (electrical current) fluxes are connected with each other, and are defined by 4 kinetic coefficients [12], having a tensor structure in presence of a magnetic field. The general consideration of heat and electrical conductivity of degenerate electrons will be done elsewhere.

The new coefficients can be used for calculation of temperature distribution in white dwarfs, on the surface and in the crust of magnetized neutron star, as well as in the magnetized matter accreting to the magnetized neutron star. The temperature distribution over the surface of NS is important for understanding of the geometry of magnetic field inside the neutron star and near its surface.

\section{ACKNOWLEDGMENTS}

The work of GSBK and MVG was supported by the Russian Science Foundation grant No. 15-12-30016.
[1] D. Page, U. Geppert, F. Weber, Nuclear Physics A 777, 497 (2006).

[2] J.A. Pons , F.M. Walter, J.M. Lattimer et al., Astrophysical Journal 564, 981 (2002).

[3] V.E. Zavlin, Astrophysical Journal 665, L143 (2007).

[4] A.V. Kuznetsov, N.V. Mikheev, Electroweak processes in the external active medium (Yaroslavl', 2010). In Russian.

[5] D.N. Aguilera, J.A. Pons, J.A. Miralles, Astronomy \&
Astrophysics 486, 271 (2008).

[6] D.G. Yakovlev, K.P. Levenfish, Yu.A. Shibanov, Uspekhi Fiz. Nauk 169, 825 (1999).

[7] E.E. Salpeter, Astrophysical Journal 134, 669 (1961).

[8] E. Flowers, N. Itoh, Astrophysical Journal 206, 218 (1976).

[9] D.G. Yakovlev, V.A. Urpin, On thermal and electrical conductivities in neutron stars and white dwarfs. Astron. 
Zh. 57, 526 (1980).

[10] N. Itoh, H. Hayashi, Y. Kohyama, Astrophysical Journal 418, 405 (1993).

[11] D.G. Yakovlev, O.Y. Gnedin, A.D. Kaminker, K.P. Levenfish, A.Y. Potekhin, Neutron star cooling: theoretical aspects and observational constraints. Advances in Space Research 33, 523 (2006).

[12] S. Chapmen and T.G. Cowling, Mathematical Theory of Nonuniform Gases (Cambrige, 1952) [Russian translation published by IL, Moscow, 1958].

[13] E. Uehling, G. Uhlenbeck, Transport Phenomena in Einstein-Bose and Fermi-Dirac Gases. I. Phys. Rev. 43, 552 (1933).

[14] E. Uehling, Transport Phenomena in Einstein-Bose and Fermi-Dirac Gases. II. Phys. Rev. 46, 917 (1934).

[15] S.Z. Tomonaga, Innere Reibung und Warmeleitfahigkeit der Kernmaterie. Z. Phys. 110, 573 (1938).

[16] G.S. Bisnovatyi-Kogan, M.M. Romanova, JETP 56, 243 (1983).

[17] V.S. Imshennik On the thermal conductivity of plasma. Astronomicheskii Zhurnal 38, 652 (1961).

[18] L.D. Landau, Journ. Exper. Theor. Phys (JETP) 7, 203 (1937). (Phys. Zeit. Sow., Bd. 10. S. 154, 1936).

[19] S. Chandrssekhar, Stochastic Problems in Physics and Astronomy. Rev. Modern Phys. 15, 1 (1943).

[20] M. Rosenbluth, W.M. Macdonald, D.L. Judd, Physical Review 107, 1 (1957).

[21] B.A. Trubnikov Particle Interactions in a Fully Ionized Plasma. Reviews of Plasma Physics, Volume 1. Edited by M. A. Leontovich, Consultants Bureau, New York, p.105 (1965).

[22] R. Landshoff, Physical Review 82, 442 (1951).

[23] W. Marshall, The kinetic theory of an ionized gas. p. 3 At. Energy Res Estable, N T/R, 2419 (1960).
[24] G.S. Bisnovatyi-Kogan, Transport properties of partially ionized two-temperature plasma and isotropic corrections to maxvellian distribution function (in Russian) (Diploma thesis, Moscow Institute of Physics and Technology, 1964).

[25] G.S. Bisnovatyi-Kogan, Journal of Applied Mechanics and Technical Physics, No. 3, p. 43 (1964).

[26] S.I. Braginskii, JETP 6, 358, 1957.

[27] N.A. Bobrova, P.V. Sasorov, Plasma Physics Reports 19, 409 (1993).

[28] A.A. Wyller, Astrophisica Norvegica 9, 79 (1964).

[29] M. Lampe, Physical Review 170, 306 (1968).

[30] A. Wiranata, M. Prakash, Physical Review C 85, 5 (2012).

[31] A. Wiranata, M. Prakash, P. Chakraborty, Central European Journal of Physics 10, 1349 (2012).

[32] L.D. Landau, E.M. Lifshitz, Statistical Physics. 5, 3rd ed. (Butterworth-Heinemann, 1980).

[33] D. Burnett, "The Distribution of Molecular Velocities and the Mean Motion in a Non-Uniform Gas," Proc. Lond. Math. Soc., 40, 382 (1936).

[34] R. Balesku, Equilibrium and Non-Equilibrium Statistical Mechanics (John Wiley \& Sons, 1975).

[35] G.S. Bisnovatyi-Kogan, Stellar Physics I: Fundamental Concepts and Stellar Equilibrium (Springer, 2001).

[36] V.L. Ginzburg, A.A. Rukhadze, Waves in Magnetoactive Plasma (M., Nauka, 1970).

[37] E. Yanke, F. Emde, F. Lösch, Tafeln Höherer Funktionen (B.G. Teubner Verlagsgesellschaft, 1960)

[38] L.E. Kalikhman, Elements of magnetohydrodynamics (Atomizdat, Moscow, 1964).

[39] E. Schatzman, White dwarfs (Amsterdam, North Holland, 1958).

[40] A.A. Wyller, Astrophysical Journal 184, 517 (1973). 